\title{
A MIXING CONDITION FOR EXTREME LEFT INVARIANT MEANS $\left({ }^{1}\right)$
}

\author{
BY \\ S. P. LLOYD
}

1. Introduction. Let the discrete semigroup $\Sigma$ be left amenable [1] and let $L M$ be the set of left invariant means for $\Sigma$. We call a bounded function $u$ on $\Sigma$ left almost convergent to value $k$ when $(u, \lambda)=k$ holds for every $\lambda \in L M$. (Similar generalizations of the almost convergence of $\mathrm{G}$. G. Lorentz have been given by Day [1] and Dye [2].) Let $V(\sigma), \sigma \in \Sigma$, be an antirepresentation of $\Sigma$ by nonnegative operators on $C(X)$, with $X$ compact Hausdorff and $V(\sigma) 1=1$. The adjoints $V^{*}(\sigma)$, $\sigma \in \Sigma$, are a representation of $\Sigma$ by nonnegative operators of unit norm on the space $r c a(X)$ of regular bounded signed Borel measures on $X$. Let $L M(X)$ be the set of probability measures in $r c a(X)$ which are fixed under each $V^{*}(\sigma), \sigma \in \Sigma$. Then $L M(X)$ is convex, weakly* compact, and nonempty, and we are concerned with properties of the extreme points of $L M(X)$. The following is one of our main results. For each $f, g \in C(X)$ and each $\mu \in L M(X)$ the function $(f \cdot V(\sigma) g, \mu)$, $\sigma \in \Sigma$, is left almost convergent to a value depending on $f, g, \mu$. For $\mu$ to be extreme in $\operatorname{LM}(X)$ it is necessary and sufficient that for each $f, g \in C(X)$ the value be $(f, \mu)(g, \mu)$. This is a mixing condition which generalizes the result of Sucheston [3] for the case $\Sigma=$ additive positive integers. Our result is distinct from that of Dye [2], who is concerned with representations of $\Sigma$ by operators on Hilbert space.

The first part of the paper is expository. In $\$ 2$ we describe results obtained by the author concerning nonnegative projections of unit norm in $C(X)$. In $\S 3$ we compile results of Arens on extending the product in Banach algebras and modules. Cases of particular interest are discussed in $\$ \$ 4$ and 5. In $\$ 6$ we obtain an algebraic characterization of the extreme points of $L M(X)$. In $\$ 7$ various projections associated with left invariant means are investigated, and in $\$ 8$ we discuss left almost convergence. In $\$ 9$ the right and two sided cases are reduced to the left case.

2. Nonnegative projections of unit norm in $C(X)$. References for the present section are [4, p. 490], [5]. Let $X$ be a compact Hausdorff space, let $X$ be the

Presented to the Society, January 24, 1966; received by the editors March 10, 1966 and, in revised form, May 23, 1966.

(1) Part of this research was done while the author was Visiting Associate Professor of Statistics at the University of Chicago. It was partially supported by the Army Research Office, Office of Naval Research, and Air Force Office of Scientific Research by Contract No. Nonr 212(23), NR342-043, and by Research Grant No. G-21058 from the Division of Mathematical, Physical, and Engineering Sciences of the National Science Foundation. 
$\sigma$-field of Borel subsets of $X$, and let $C(X)$ be the continuous real functions on $X$. We represent the Banach space conjugate of $C(X)$ as the space $r c a(X)$ of regular bounded signed Borel measures on $X$. By $r c a(X) \backslash C(X)$ we will mean $r c a(X)$ in its $C(X)$ topology, and similarly for other linear spaces. We denote by $r p(X)$ the set of probability measures in $r c a(X)$. We denote by $\delta_{x} \in r p(X)$ the unit point measure at $x \in X$, i.e., evaluation at $x$. Sometimes we will deliberately confuse $x$ and $\delta_{x}$, choosing to regard $X$ as a subset of $r c a(X) \mid C(X)$.

Let $T: C(X) \rightarrow C(X)$ be a bounded linear operator on $C(X)$, with adjoint $T^{*}: r c a(X) \rightarrow r c a(X)$, and let $t: X \rightarrow r c a(X)$ be the function defined by $t_{x}=T^{*} \delta_{x}$, $x \in X$. Then $t$ has the properties

$$
\begin{aligned}
& t: X \rightarrow \operatorname{rca}(X) \mid C(X) \text { is continuous, } \\
& \|T\|=\sup _{x \in X}\left\|t_{x}\right\|, \\
& T \geqq 0 \text { iff } t_{x} \geqq 0 \text { for all } x \in X,
\end{aligned}
$$

and $T$ has the representation

$$
T f(x)=\int f\left(x^{\prime}\right) t_{x}\left(d x^{\prime}\right), \quad x \in X, f \in C(X) .
$$

We will be concerned with nonnegative projections of unit norm in $C(X)$. Let $\chi_{A}$ be the characteristic function of set $A$. Our first result is

THEOREM 1. Let $T$ be a bounded nonnegative projection in $C(X)$ such that the complementary projection $1-T$ is also nonnegative. Then there exists an open closed subset $A$ of $X$ such that $T f(x)=\chi_{A}(x) f(x), x \in X, f \in C(X)$.

Proof. From

$$
\begin{aligned}
T f(x) & =\int f\left(x^{\prime}\right) t_{x}\left(d x^{\prime}\right), \\
(1-T) f(x) & =\int f\left(x^{\prime}\right)\left[\delta_{x}\left(d x^{\prime}\right)-t_{x}\left(d x^{\prime}\right)\right],
\end{aligned}
$$

it is easy to see that if both $T$ and $1-T$ are to be nonnegative then for each $x \in X$ the closed support of the nonnegative measure $t_{x}$ cannot extend beyond the singleton set $\{x\}$. From $T^{2}=T$ there follows $\left[t_{x}(\{x\})\right]^{2}=t_{x}(\{x\})$, and set $A$ of the theorem is $A=\left\{x: t_{x}(\{x\})=1\right\}$. The continuity of $(T 1)(x)=t_{x}(X)=t_{x}(\{x\}), x \in X$, insures that $A$ is open closed.

More generally, suppose that $T$ is a nonnegative projection of unit norm in $C(X)$. Let $Y$ denote the range of $t$ in $r c a(X) \mid C(X)$, and let $Y_{o}$ be the set of nonzero extreme points of the closed convex hull of $Y \cup\{0\}$ in $r c a(X) \mid C(X)$. Then $Y_{o}$ is a nonempty closed subset of $Y$, and $t$ has the further properties

(1) If $t_{x}=y \in Y_{o}$ then $t_{x}$ is a probability measure with closed support contained in $t^{-1} y\left(=\left\{x: t_{x}=y\right\}\right)$. 
(2) If $x \in X$ then $t_{x}$ is a nonnegative measure whose closed support is contained in $t^{-1} Y_{o}$, and such that $t_{x}(X) \leqq 1$.

The set $Y_{o}$ is the Choquet boundary [6] of range $T$; the existence of a projection as above insures that it is closed. We call $\mathscr{S}(T)=t^{-1} Y_{o}$ the support of $T$; each measure in range $T^{*}$ has closed support contained in $\mathscr{S}(T)$.

From the representation we obtain a weak averaging identity, as follows.

THEOREM 2. Suppose $T$ is a nonnegative projection of unit norm in $C(X)$. Then

$$
T(f \cdot T g)=T(T f \cdot T g) \quad \text { for all } f, g \in C(X) .
$$

Proof. When $x \in \mathscr{S}(T)$ the measures $t_{x}$ are probabilities localized in equivalence classes $t^{-1} y$, and since $T g$ is constant on equivalence classes the averaging identity $T(f \cdot T g)=T f \cdot T g$ holds on $\mathscr{S}(T)$. Applying $T$ and using (2), we obtain the theorem.

In the case that range $T$ is a subalgebra of $C(X)$ there obtains $T(T f \cdot T g)=T f \cdot T g$, and (3) becomes the averaging identity. Even when range $T$ is not a subalgebra of $C(X)$ we can introduce an algebraic structure into range $T$, as follows. For $f, g \in C(X)$ let the product of $T f$ and $T g$ in range $T$ be defined as $T(T f \cdot T g)$, where in the parentheses the $C(X)$ (pointwise) product is intended. Use of (3) shows that this is an associative operation, and with this product and supremum norm range $T$ becomes an algebra isometrically algebraically isomorphic to $C\left(Y_{o}\right)$. Suppose $\lambda \in \operatorname{rp}(X)$ induces a homomorphism of range $T$ onto the reals, that is,

$$
(T(T f \cdot T g), \lambda)=(T f, \lambda)(T g, \lambda)
$$

for all $f, g \in C(X)$ and $(T 1, \lambda)=1$. Then the closed support of $\lambda$ must be contained in some equivalence class $t^{-1} y$ for some $y \in Y_{o}$, and $T^{*} \lambda=y$ is the element of range $T^{*}$ inducing the same homomorphism. Using Theorem 2, we see that if $\lambda \in$ range $T^{*}$ then for $\lambda \in Y_{o}$ to hold it is necessary and sufficient that $\lambda \neq 0$ and $(f \cdot T g, \lambda)=(f, \lambda)(g, \lambda)$ for all $f, g \in C(X)$.

If $f \in C(X), \lambda \in r c a(X)$, then $f \lambda \in r c a(X)$ will denote the measure with RadonNikodym derivative $d(f \lambda) / d \lambda=f$.

Theorem 3. Suppose $\lambda \in r c a(X)$ is in range $T^{*}, T^{*} \lambda=\lambda$. Then $T^{*}(f \lambda)=(T f) \lambda$ holds for all $f \in C(X)$.

Proof. For all $g \in C(X)$ we have

$$
\begin{aligned}
\left(g, T^{*}(f \lambda)\right) & =(T g, f \lambda)=(f \cdot T g, \lambda) \\
& =(T(f \cdot T g), \lambda)=(T(T f \cdot T g), \lambda) \\
& =(T(T f \cdot g), \lambda) \\
& =(T f \cdot g, \lambda)=(g,(T f) \lambda),
\end{aligned}
$$

where at various places we have used (3), $T^{*} \lambda=\lambda$, and the Radon-Nikodym theorem. 
3. Arens products. References for the present section are [7], [8], [9]. Let $\mathscr{X}$ be a Banach algebra with conjugate space $\mathscr{X}^{*}$, second conjugate space $\mathscr{X}^{* *}$, and let $\kappa: \mathscr{X} \rightarrow \mathscr{X}^{* *}$ be the natural embedding. Since $\kappa \mathscr{X}$ is dense in $\mathscr{X}^{* *} \mid \mathscr{X}^{*}$, the algebraic product in $\mathscr{X}$ lifts in the obvious way to a product on the dense subset $\kappa \mathscr{X}$ of $\mathscr{X}^{* *} \mid \mathscr{X}^{*}$. As it turns out, the product can be extended by continuity to all of $\mathscr{X}^{* *}$, in at least two ways.

Suppose $\left\{x_{\alpha}\right\}$ is a generalized Cauchy sequence in $\mathscr{X} \mid \mathscr{X}^{*}$ such that $\left\{\kappa x_{\alpha}\right\}$ converges in $\mathscr{X}^{* *} \mid \mathscr{X}^{*}$ to $u \in \mathscr{X}^{* *}$, and let $\left\{\kappa y_{\beta}\right\}$ converge similarly to $v \in \mathscr{X}^{* *}$. Put

$$
\begin{aligned}
& u\langle\mathfrak{l}\rangle v^{\prime}=\lim _{\alpha}\left(\lim _{\beta} \kappa\left(x_{\alpha} y_{\beta}\right)\right), \\
& u\langle\mathfrak{r}\rangle v=\lim _{\beta}\left(\lim _{\alpha} \kappa\left(x_{\alpha} y_{\beta}\right)\right) .
\end{aligned}
$$

Then each of $\langle\mathfrak{l}\rangle,\langle\mathfrak{r}\rangle$ is an associative product in $\mathscr{X}^{* *}$ which makes it a Banach algebra and which reduces to the given product on $\kappa \mathscr{X}$. (We remark that $\langle\mathfrak{l}\rangle$ is the usual Arens product.)

We will not give the proof that this heuristic formulation makes sense. It would require all the steps of the Arens construction, which we now reproduce. The notational device is essentially that of Gulick [10]. For any Banach space $\mathscr{Z}$, $(z, \zeta)$ will denote the value of $\zeta \in \mathscr{Z} *$ at $z \in \mathscr{Z}$. In each of the following, the lefthand side serves to define the operation introduced in the right-hand side:

$$
\begin{aligned}
(x y, \xi) & =(x, y<\mathfrak{r}>\xi), & & x \in \mathscr{X}, y \in \mathscr{X}, \xi \in \mathscr{X}^{*}, \\
(y<\mathfrak{r}>\xi, u) & =(y, \xi\langle\mathfrak{x}>u), & & y \in \mathscr{X}, \xi \in \mathscr{X}^{*}, u \in \mathscr{X}^{* *}, \\
(\xi\langle\mathfrak{r}>u, v) & =(\xi, u\langle\mathfrak{r}>v), & & \xi \in \mathscr{X}^{*}, u \in \mathscr{X}^{* *}, v \in \mathscr{X}^{* *} .
\end{aligned}
$$

Similarly,

$$
\begin{aligned}
(x y, \xi) & =(y, \xi\langle\mathfrak{l}>x), & & x \in \mathscr{X}, y \in \mathscr{X}, \xi \in \mathscr{X}^{*}, \\
(\xi\langle\mathfrak{l}>x, v) & =(x, v\langle\mathfrak{l}>\xi), & & x \in \mathscr{X}, \xi \in \mathscr{X}^{*}, v \in \mathscr{X}^{* *}, \\
(v\langle\mathfrak{l}\rangle \xi, u) & =(\xi, u\langle\mathfrak{l}>v), & & \xi \in \mathscr{X}^{*}, u \in \mathscr{X}^{* *}, v \in \mathscr{X}^{* *}
\end{aligned}
$$

For each of the operations $\langle\mathfrak{l}\rangle$ there is weak* continuity in the left-hand factor for fixed right-hand factor, as well as separate weak and joint norm continuity in both factors. Each $\langle\mathfrak{r}\rangle$ is (separately) weakly* continuous in the right-hand factor. If $u, v \in \mathscr{X}^{* *}$ and either of $u$ or $v$ is an element of $\kappa \mathscr{X}$ then $u\langle\mathfrak{l}\rangle v=u\langle\mathfrak{r}\rangle v$, and for this we will use the notation $u \ominus v$ when useful.

The following generalization, also due to Arens, will be needed. Let $\mathscr{X}$ be a Banach algebra, and let Banach space $\mathscr{Y}$ be a Banach right module over $\mathscr{X}$. That is, for each $y \in \mathscr{Y}, x \in \mathscr{X}$ a bilinear product $y x \in \mathscr{Y}$ is defined with the property $\left(y x_{1}\right) x_{2}=y\left(x_{1} x_{2}\right)$, and $\|y x\| \leqq M\|y\|\|x\|$ holds for some $0 \leqq M<\infty$. Then $y x=V(x) y$ serves to define $V(x): \mathscr{Y} \rightarrow \mathscr{Y}$ as a norm continuous antirepresentation of $\mathscr{X}$ by bounded linear operators on $\mathscr{Y}$. That is, $V(x)$ depends linearly on $x \in \mathscr{X}$, and $V\left(x_{1}\right) V\left(x_{2}\right)=V\left(x_{2} x_{1}\right),\|V(x)\| \leqq M\|x\|$. Conversely, if such an antirepresentation is 
given then a right module structure is determined. In each of the following, the left-hand side serves to define the operation introduced in the right-hand side:

$$
\begin{aligned}
(y x, \eta) & =(x, \eta\langle\mathfrak{l}\rangle y), & & y \in \mathscr{Y}, x \in \mathscr{X}, \eta \in \mathscr{Y}^{*}, \\
(\eta\langle\dot{\mathfrak{l}}\rangle y, u) & =(y, u\langle\mathfrak{l}\rangle \eta), & & \eta \in \mathscr{Y}^{*}, y \in \mathscr{Y}, u \in \mathscr{X}^{* *}, \\
(u\langle\mathfrak{l}\rangle \eta, v) & =(\eta, v\langle\mathfrak{l}\rangle u), & & u \in \mathscr{X}^{* *}, \eta \in \mathscr{Y}^{*}, v \in \mathscr{Y}^{* *} .
\end{aligned}
$$

With $\langle\mathfrak{l}\rangle$ operations as in (5), we have the association rules

$$
\begin{aligned}
\eta\langle\dot{\mathfrak{l}}\rangle(y x) & =(\eta\langle\dot{\mathfrak{l}}\rangle y)\langle\mathfrak{l}\rangle x, \\
(u\langle\dot{\mathfrak{l}}\rangle \eta)\langle\dot{\mathfrak{l}}\rangle y & =u\langle\mathfrak{l}\rangle(\eta\langle\dot{\mathfrak{l}}\rangle y), \\
u_{1}\langle\dot{\mathfrak{l}}\rangle\left(u_{2}\langle\dot{\mathfrak{l}}\rangle \eta\right) & =\left(u_{1}\langle\mathfrak{l}\rangle u_{2}\right)\langle\dot{\mathfrak{l}}\rangle \eta, \\
\left(v\langle\mathfrak{l}\rangle u_{1}\right)\langle\dot{\mathfrak{l}}\rangle u_{2} & =v\langle\dot{\mathfrak{l}}\rangle\left(u_{1}\langle\mathfrak{l}\rangle u_{2}\right),
\end{aligned}
$$

for $x \in \mathscr{X}, y \in \mathscr{Y}, \eta \in \mathscr{Y}^{*}, u, u_{1}, u_{2} \in \mathscr{X}^{* *}, v \in \mathscr{Y}^{* *}$.

The adjoint $V^{*}(x): \mathscr{Y}^{*} \rightarrow \mathscr{Y}^{*}$ is a representation of $\mathscr{X}$, and $V^{*}(x) \eta=\kappa x\langle\dot{\mathfrak{l}}\rangle \eta$, $x \in \mathscr{X}, \eta \in \mathscr{Y}^{*}$, in terms of the Arens operations defined above. Let $\left\{x_{\alpha}\right\}$ be a generalized sequence in $\mathscr{X}$ such that $\left\{\kappa x_{\alpha}\right\}$ converges in $\mathscr{X}^{* *} \mid \mathscr{X}^{*}$ to $u \in \mathscr{X}^{* *}$, so that $\left\{\kappa x_{\alpha}\langle\dot{\mathfrak{l}}\rangle \eta\right\}$ converges in $\mathscr{Y}^{*} \mid \mathscr{Y}$ to $u\langle\dot{\mathfrak{l}}\rangle \eta$. If for $u \in \mathscr{X}^{* *}$ we define $Q(u): \mathscr{Y}^{*} \rightarrow \mathscr{Y}^{*}$ by $Q(u) \eta=u\langle\mathfrak{i}\rangle \eta, \eta \in \mathscr{Y}^{*}$, then $Q(u)=\lim V^{*}\left(x_{\alpha}\right)$ in the weak* operator topology. It is easy to verify from (7) that $Q\left(u_{1}\langle\mathfrak{l}\rangle u_{2}\right)=Q\left(u_{1}\right) Q\left(u_{2}\right), u_{1}, u_{2} \in \mathscr{X}^{* *}$, whence $Q$ is an extension of the representation $V^{*}$ of $\mathscr{X}$ to the algebra $\left(\mathscr{X}^{* *},\langle\mathfrak{l}\rangle\right)$.

We mention in passing that the same right module admits Arens operations $\langle\dot{\mathfrak{r}}\rangle$ corresponding to (4), and that a Banach left module admits both $\langle\dot{\mathfrak{l}}\rangle$ and $\langle\dot{\mathfrak{r}}\rangle$ operations, so that the operations (6) and association rules (7) constitute one set out of four; (6)-(7) are the only ones we need.

4. The second conjugate of $C(X)$. References for the present section are [7], [11]. The two Arens products in $C^{* *}(X)$ are commutative and equal, and $C^{* *}(X)$ is isometrically algebraically isomorphic to $C(\alpha X)$ for a certain compact Hausdorff space $\alpha X$. We drop the $\langle\mathfrak{l}\rangle-\langle\mathrm{r}\rangle$ notation. We represent $C^{* * *}(X)$ as the space $r c a(\alpha X)$ of regular Borel measures on $\alpha X$. Let $\kappa: C(X) \rightarrow C(\alpha X)$ and $\bar{\kappa}: r c a(X) \rightarrow r c a(\alpha X)$ be the natural embeddings. The restriction of $\kappa^{*}: r c a(\alpha X) \rightarrow r c a(X)$ to the unit point measures induces a continuous epimorphism $\pi: \alpha X \rightarrow X$. Under $\pi, \alpha X$ partitions into equivalence classes $\pi^{-1} x, x \in X$, and $\kappa C(X)$ are the elements of $C(\alpha X)$ constant on $\pi$ equivalence classes. If $\theta \in r c a(\alpha X)$ is a measure on $\alpha X$ then $\kappa^{*} \theta$ is the measure on $X$ given by $\left(\kappa^{*} \theta\right)(E)=\theta\left(\pi^{-1} E\right), E \in X$. That is, one simply restricts the domain of $\theta$ to unions of $\pi$ equivalence classes.

The space $\alpha X$ is hyperstonian, and $\bar{\kappa} r c a(X)$ are the normal measures for $\alpha X$. That is, $\alpha X$ is extremally disconnected, the closed support of each $\bar{\kappa} \lambda, \lambda \in \operatorname{rca}(X)$, is open (equivalently, $\bar{\kappa} \lambda$ vanishes on nowhere dense sets), and the union of such supports is dense in $\alpha X$. The open closed sets in $\alpha X$ correspond 1-1 to closed order ideal subspaces of $r c a(X)$. Let $M$ be such a subspace, that is, $M$ is norm closed, and 
if $\lambda \in M$ then $\nu \in M$ for all $\nu \in r c a(X)$ such that $-|\lambda| \leqq \nu \leqq|\lambda|$. Let $M^{\prime}$ be the lattice complement of $M$. Then each $\lambda \in \operatorname{rca}(X)$ has a unique decomposition $\lambda=T \lambda$ $+(1-T) \lambda$ with $T \lambda \in M,(1-T) \lambda \in M^{\prime}$, and $T, 1-T$ are complementary nonnegative projections in $r c a(X)$. Their adjoints are complementary nonnegative projections in $C(\alpha X)$, and the open closed set in $\alpha X$ associated with the decomposition $M \oplus M^{\prime}$ is the one given by Theorem 1. Conversely, if $A$ is open closed in $\alpha X$ then for $\lambda \in \operatorname{rca}(X)$ the parts of $\bar{\kappa} \lambda$ with support in $A, A^{\prime}$ are normal; it is not hard to show that this induces a decomposition of $r c a(X)$ into complementary closed order ideal subspaces.

Several types of decomposition are of interest. Let $l(X)$ be the purely atomic measures on $X$, that is, $l(X)$ is the norm closed span of $\left\{\delta_{x}: x \in X\right\}$. Denote $\left\{\bar{\kappa} \delta_{x}: x \in X\right\}$ by $\dot{X}$. Each element of $\dot{X}$ is an open point of $\alpha X$ in $r c a(\alpha X) \mid C(\alpha X)$, and the closure of $\dot{X}$ in $\alpha X$ is open in $\alpha X$ and is the Stone-Čech compactification $\beta \dot{X}$ of the discrete space $\dot{X}$. The atomic measures $l(X)$ embed under $\bar{\kappa}$ as the atomic measures with support in $\dot{X}$; the atomless measures on $X$ (if any) embed as normal measures with support contained in the open closed set $\alpha X-\beta \dot{X}$. The embedding is easy to undo, since $\kappa^{*} \bar{\kappa}=1$.

Let $\lambda \in r c a(X)$ be fixed. The $\lambda$-continuous and the $\lambda$-singular measures constitute complementary closed order ideal subspaces of $r c a(X)$. Let $\mathscr{S}_{\lambda}$ be the open closed support of $\bar{\kappa} \lambda$. Then $\mu$ is $\lambda$-continuous if $\mathscr{S}_{\mu} \subset \mathscr{S}_{\lambda}$, while $\mu$ is $\lambda$-singular if $\mathscr{S}_{\mu}$ and $\mathscr{S}_{\lambda}$ are disjoint.

Let $E \in \boldsymbol{X}$ be fixed. The measures supported by (not necessarily closed) $E$ are $\left\{\lambda \in \operatorname{rca}(X):|\lambda|\left(E^{\prime}\right)=0\right\}$, and this is a closed order ideal subspace. The corresponding open closed set in $\alpha X$ will be denoted by $E^{\wedge}$. The correspondence $E \leftrightarrow E^{\wedge}$ preserves finite set operations, and $\lambda(E)=(\bar{\kappa} \lambda)\left(E^{\wedge}\right)$ holds for all $\lambda \in r c a(X), E \in X$.

The norm closed linear subspace of $C(\alpha X)$ spanned by $\left\{\chi_{E^{\wedge}}: E \in X\right\}$ is a subalgebra isomorphic to the algebra $B(X, X)$ of bounded Borel functions on $X$, pointwise operations and supremum norm. If $\iota: B(X, X) \rightarrow C(\alpha X)$ is the embedding determined by $\iota \chi_{E}=\chi_{E^{\wedge}}, E \in X$, then $\imath^{*}: r c a(\alpha X) \rightarrow b a(X, X)$ sends measures on $\alpha X$ into finitely additive set functions on $\boldsymbol{X}$ according to $\left(\imath^{*} \theta\right)(E)=\theta\left(E^{\wedge}\right), E \in X$, $\theta \in r c a(\alpha X)$, and maps $\alpha X$ in $r c a(\alpha X) \mid C(\alpha X)$ onto (Gelfand space of $B(X, X)$ ) $=($ Boolean space of $X)$.

If $f \in C(X), \lambda \in r c a(X)$, then the Arens product $f \lambda$ is the measure on $X$ with Radon-Nikodym derivative $d(f \lambda) / d \lambda=f$. (We have already used the notation in §2.) If $\lambda \in \operatorname{rca}(X), F \in C(\alpha X)$, then the Arens product $F \lambda$ is a measure on $X$, but the derivative situation is not quite so simple. First, $\vec{\kappa}(F \lambda)$ has derivative $d \bar{\kappa}(F \lambda) / d \bar{\kappa} \lambda=F$. Let $F$ be fixed. Then for each $\lambda \in r c a(X)$ there is a bounded Borel function $\phi_{\lambda}$ on $X$ such that $d(F \lambda) / d \lambda=\phi_{\lambda}$ a.e. [ $\left.\lambda\right]$, and $\iota \phi_{\lambda}=F$ a.e. $[\bar{\kappa} \lambda]$. However, no one function on $X$ will represent $F$ for all choices of $\lambda ; B(X, X)$ is a proper subalgebra of $C(\alpha X)$, in general. Specifically, when there are atomless measures then the sets $\left\{E^{\wedge}-\beta \dot{X}: E \in X\right\}$ are not a base for the topology of $\alpha X-\beta \dot{X}$. This 
is shown by the following result, which implies that $\mathscr{S}_{\lambda}$ for $\lambda$ atomless is never of the form $E^{\wedge}-\beta \dot{X}, E \in \boldsymbol{X}$.

TheOREM 4. Suppose $\lambda \in \operatorname{rca}(X)$ is atomless and $E \in X$ is such that $|\lambda|(E) \neq 0$. Then there exist $\lambda$-singular atomless probabilities $\mu \in \operatorname{rp}(X)$ such that $\mu(E)=1$.

Proof. We may as well assume $\lambda \in r p(X)$ and $\lambda(E)=1$. By a theorem of Liapounoff we can find disjoint closed subsets $E_{0}$ and $E_{1}$ of $E$ such that $\lambda\left(E_{0}\right)=\lambda\left(E_{1}\right)$ $=1 / 3$ [12]. Let $\mathscr{C}_{1}$ be the set consisting of all $\mu \in \operatorname{rp}(X)$ such that $\mu\left(E_{0}\right)=\mu\left(E_{1}\right)$ $=1 / 2$. It is not hard to show that $\mathscr{C}_{1}$ is nonempty and compact in $r c a(X) \mid C(X)$. Now choose disjoint closed sets $E_{00}, E_{01} \subset E_{0}$ and $E_{10}, E_{11} \subset E_{1}$ such that $\lambda\left(E_{i j}\right)=1 / 9$ each, and let $\mathscr{C}_{2}$ consist of all $\mu \in \operatorname{rp}(X)$ such that $\mu\left(E_{i j}\right)=1 / 4$ each. Again, $\mathscr{C}_{2}$ is nonempty and compact in $r c a(X) \mid C(X)$; moreover, $\mathscr{C}_{2} \subset \mathscr{C}_{1}$. Continuing the Cantor type construction, we obtain a nest $\mathscr{C}_{1} \supset \mathscr{C}_{2} \supset \cdots$ of nonempty compact sets. It should be clear that any element of nonempty $\bigcap_{1}^{\infty} \mathscr{C}_{n}$ is an atomless probability measure, is $\lambda$-singular, and has support in $E$.

A similar result holds for the universal completion of $\boldsymbol{X}$. That is, for each $\lambda \in \operatorname{rca}(X)$ let $\boldsymbol{X}_{\lambda}$ be the completion of $\boldsymbol{X}$ with respect to the outer measure induced by $(X,|\lambda|)$, and define $\boldsymbol{X}_{c}=\bigcap_{\lambda} \boldsymbol{X}_{\lambda}$. To each $E \in \boldsymbol{X}_{c}$ there corresponds open closed $E^{\wedge} \subset \alpha X$. Again, $\left\{E^{\wedge}-\beta \dot{X}: E \in X_{c}\right\}$ is not a base for $\alpha X-\beta \dot{X}$. We make no further use of $\boldsymbol{X}_{c}$.

5. The conjugates of $c_{0}(\Sigma)$. Let $\Sigma$ be a discrete space, and let $c_{0}(\Sigma)$ be the Banach algebra of bounded real functions vanishing at infinity, with pointwise operations and supremum norm. Some successive Banach space conjugates of $c_{o}(\Sigma)$ are

$$
\begin{aligned}
& c_{o}(\Sigma), \\
& l(\Sigma), \\
& m(\Sigma) \text { or } C(\beta \Sigma), \\
& b a(\Sigma) \text { or } \quad r c a(\beta \Sigma), \\
& C(A) \text { with } A=\alpha(\beta \Sigma), \\
& r c a(A) .
\end{aligned}
$$

Here, $l(\Sigma)$ is the space of purely atomic measures on the field of all subsets of $\Sigma, m(\Sigma)$ is the algebra of bounded functions on $\Sigma$, isomorphic to $C(\beta \Sigma)$ with $\beta \Sigma$ the Stone-Čech compactification of $\Sigma, b a(\Sigma)$ is the space of bounded finitely additive set functions on subsets of $\Sigma$, isomorphic to the regular Borel measures on $\beta \Sigma$, and $A$ is the Arens space $\alpha X$ described in the preceding section with $X=\beta \Sigma$.

Let us catalog the natural embeddings and their adjoints.

(i) $\kappa_{0}: c_{0}(\Sigma) \rightarrow C(\beta \Sigma)$. A function vanishing at infinity extends to 0 on $\beta \Sigma-\Sigma$, so $\kappa_{0} c_{0}(\Sigma)$ is an ideal.

(ii) $\kappa_{1}: l(\Sigma) \rightarrow r c a(\beta \Sigma)$. The unit point measure $\delta_{\sigma} \in l(\Sigma)$ embeds as the unit point measure at $\sigma \in \Sigma \subset \beta \Sigma$; we denote $\kappa_{1} \delta_{\sigma}$ simply by $\delta_{\sigma}$.

(iii) $\kappa_{2}: C(\beta \Sigma) \rightarrow C(A)$ as in $\S 4$. 
(iv) $\kappa_{3}: r c a(\beta \Sigma) \rightarrow r c a(A)$ as in $\$ 4$.

(v) $\kappa_{0}^{*}: \operatorname{rca}(\beta \Sigma) \rightarrow l(\Sigma)$ picks out the purely atomic part of the measure living on $\Sigma$ in $\beta \Sigma . \kappa_{0}^{*}(\beta \Sigma)$ is the one point compactification of $\Sigma$, with 0 as the point at infinity.

(vi) $\kappa_{1}^{*}: C(A) \rightarrow C(\beta \Sigma)$. The closure of $\kappa_{3} \Sigma$ in $A$ is open in $A$ and a copy of $\beta \Sigma ; \kappa_{1}^{*}$ is restriction of domain to $\left(\kappa_{3} \Sigma\right)^{-}$.

(vii) $\kappa_{2}^{*}: r c a(A) \rightarrow r c a(\beta \Sigma)$ as in $\$ 4$.

(viii) $\kappa_{0}^{* *}: C(\beta \Sigma) \rightarrow C(A)$ copies the given function on $\left(\kappa_{3} \Sigma\right)^{-}$and puts 0 elsewhere.

(ix) $\kappa_{1}^{* *}: r c a(\beta \Sigma) \rightarrow r c a(A)$ copies the given measure on $\left(\kappa_{3} \Sigma\right)^{-}=\kappa_{1}^{* *}(\beta \Sigma)$.

(x) $\kappa_{0}^{* * *}: r c a(A) \rightarrow r c a(\beta \Sigma)$ picks out the part of the measure living on $\left(\kappa_{3} \Sigma\right)^{-}$.

Suppose now that $\Sigma$ is a semigroup. The unit point measures $\left\{\delta_{\sigma}: \sigma \in \Sigma\right\}$ are a basis for $l(\Sigma)$, and the familiar convolution product in $l(\Sigma)$ is determined by the basis values $\delta_{\sigma} * \delta_{\tau}=\delta_{\sigma \tau}, \sigma, \tau \in \Sigma$. This product has Arens extensions $\langle\mathfrak{l}\rangle$ and $\langle\mathfrak{r}\rangle$ in $\operatorname{rca}(\beta \Sigma)$. The restriction of these to the unit point measures makes $\beta \Sigma$ a (nontopological) semigroup in two ways; $x\langle\mathfrak{l}\rangle y$ is continuous in $x$ while $x\langle\mathfrak{r}\rangle y$ is continuous in $y, x, y \in \beta \Sigma$.

We remark that $\langle\mathfrak{l}\rangle$ and $\langle\mathfrak{r}\rangle$ in $r c a(\beta \Sigma)$ each have two Arens extensions in $r c a(A)$, so that there are four products of interest in $\operatorname{rca}(A)$. We will not need any of these explicitly.

For each $s \in l(\Sigma)$ the left regular representation $L(s): l(\Sigma) \rightarrow l(\Sigma)$ is defined by $L(s) t=s * t, t \in l(\Sigma)$. This is a nonnegative norm continuous representation of $l(\Sigma)$; that is, $L\left(s_{1}\right) L\left(s_{2}\right)=L\left(s_{1} * s_{2}\right), L(s) \geqq 0$ if $s \geqq 0,\|L(s)\| \leqq\|s\|$. The restriction $L\left(\delta_{\sigma}\right)$, $\sigma \in \Sigma$, to the unit point measures we denote by $L(\sigma)$, and have $L\left(\sigma_{1}\right) L\left(\sigma_{2}\right)=L\left(\sigma_{1} \sigma_{2}\right)$, $L(\sigma) \geqq 0,\|L(\sigma)\|=1$.

The right regular antirepresentation $R(s): l(\Sigma) \rightarrow l(\Sigma)$ is defined similarly by $R(s) t=t * s, t \in l(\Sigma)$, and for the restriction to $\Sigma$ we obtain an antirepresentation:

$$
R\left(\sigma_{1}\right) R\left(\sigma_{2}\right)=R\left(\sigma_{2} \sigma_{1}\right), R(\sigma) \geqq 0,\|R(\sigma)\|=1 .
$$

The adjoints of these operations can be expressed in terms of the Arens extensions of the convolution product in $l(\Sigma)$. The following catalog will prove useful.

$$
\begin{aligned}
& L(\sigma) t=\delta_{\sigma} * t \quad \text { (rep.), } \\
& R(\sigma) t=t * \delta_{\sigma} \quad \text { (antirep.), } \\
& t \in l(\Sigma), \\
& \left(L^{*}(\sigma) f\right)(x)=f(\sigma \ominus x)=\left(f \ominus \delta_{\sigma}\right)(x) \quad \text { (antirep.), } \\
& \left(R^{*}(\sigma) f\right)(x)=f(x \ominus \sigma)=\left(\delta_{\sigma} \ominus f\right)(x) \quad \text { (rep.), } \\
& x \in \beta \Sigma, \quad f \in C(\beta \Sigma), \\
& L^{* *}(\sigma) \mu=\delta_{\sigma} \ominus \mu \quad \text { (rep.), } \\
& R^{* *}(\sigma) \mu=\mu \ominus \delta_{\sigma}, \quad \mu \in \operatorname{rca}(\beta \Sigma) \quad \text { (antirep.). }
\end{aligned}
$$


6. Left invariant means. A fixed vector of a representation of $\Sigma$ will be called left invariant, a fixed vector of an antirepresentation being right invariant. The particular representation $L^{* *}(\sigma)$ of $\S 5$ acts on $r c a(\beta \Sigma)$, and the left invariant elements of $r p(\beta \Sigma)$ are the left invariant means for $\Sigma$. We assume in all that follows that $\Sigma$ is left amenable, and we denote by $L M$ the set of left invariant means. A left invariant mean is a mean $\lambda \in \operatorname{rp}(\beta \Sigma)$ such that $\delta_{\sigma}\langle\mathfrak{l}\rangle \lambda=L^{* *}(\sigma) \lambda=\lambda, \sigma \in \Sigma$; by weak* continuity this is equivalent to $\mu\langle\mathfrak{l}\rangle \lambda=\lambda$ for all $\mu \in \operatorname{rp}(\beta \Sigma)$.

Suppose $V(\sigma): \mathscr{Y} \rightarrow \mathscr{Y}$ is a bounded antirepresentation of $\Sigma$ by operators on a Banach space $\mathscr{Y}$. That is, $V\left(\sigma_{1}\right) V\left(\sigma_{2}\right)=V\left(\sigma_{2} \sigma_{1}\right)$ and $\|V(\sigma)\| \leqq M$. If $s=\sum_{\sigma} s_{\sigma} \delta_{\sigma} \in l(\Sigma)$ put $V(s)=\sum_{\sigma} s_{\sigma} V(\sigma)$, making $V(s), s \in l(\Sigma)$, a norm continuous antirepresentation of $l(\Sigma)$. With the product $y * s=\sum_{\sigma} s_{\sigma} V(\sigma) y, \mathscr{Y}$ becomes a Banach right module over $l(\Sigma)$.

The association rules (7) show that $\mathscr{Y}^{*}$ is a Banach left module over $(\operatorname{rca}(\beta \Sigma),\langle\mathfrak{l}\rangle)$. Let $Q(\mu), \mu \in r c a(\beta \Sigma)$, be the extension of $V^{*}(s), s \in l(\Sigma)$, as in $\S 3$. Suppose $\lambda \in L M$ is a left invariant mean, and consider the operator $Q(\lambda): \mathscr{Y}^{*} \rightarrow \mathscr{Y}^{*}$. From

$$
V^{*}(\sigma) Q(\lambda)=Q\left(\delta_{\sigma}\right) Q(\lambda)=Q\left(\delta_{\sigma}\langle\mathfrak{l}\rangle \lambda\right)=Q(\lambda),
$$

$\sigma \in \Sigma$, it follows that every vector $Q(\lambda) \eta=\lambda\langle\dot{\mathfrak{l}}\rangle \eta$ in range $Q(\lambda)$ is left invariant for the representation $V^{*}(\sigma)$. On the other hand, suppose $\eta \in \mathscr{Y}^{*}$ is left invariant. Let $\left\{\lambda_{\alpha}\right\}$ be a generalized sequence of means in $l(\Sigma)$ such that $\left\{\kappa_{1} \lambda_{\alpha}\right\}$ converges in $\operatorname{rca}(\beta \Sigma) \mid C(\beta \Sigma)$ to $\lambda \in L M$. From $V^{*}(\sigma) \eta=\eta$ there follows $V^{*}\left(\lambda_{\alpha}\right) \eta=\eta$ and hence $Q(\lambda) \eta=\lim V^{*}\left(\lambda_{\alpha}\right) \eta=\eta$. Thus $Q(\lambda)$ is a projection onto the left invariant vectors of the representation $V^{*}(\sigma)$ on $\mathscr{Y}^{*}$. From $\left\|V^{*}(\sigma)\right\| \leqq M$ there follows $\|Q(\lambda)\| \leqq M$. Since $\lambda_{2}\langle\mathfrak{l}\rangle \lambda_{1}=\lambda_{1}$ for $\lambda_{1}, \lambda_{2} \in L M$, one has $Q\left(\lambda_{2}\right) Q\left(\lambda_{1}\right)=Q\left(\lambda_{1}\right)$. (The above is essentially the proof of Day [13] applied to the case at hand.)

From now on we will be entirely concerned with the case where $\mathscr{Y}$ above is the space $C(X)$ for some compact Hausdorff space $X$. We assume that an antirepresentation $V(\sigma): C(X) \rightarrow C(X)$ is given with the properties $V(\sigma) \geqq 0, V(\sigma) 1=1$, whence $\|V(\sigma)\|=1, \sigma \in \Sigma$. In other words, we have a family of transition probabilities $v^{(\sigma)}: X \rightarrow r p(X)$ such that

$$
\begin{aligned}
(V(\sigma) f)(x) & =\int f\left(x^{\prime}\right) v_{x}^{(\sigma)}\left(d x^{\prime}\right), & & x \in X, f \in C(X), \\
v_{x}^{(\sigma)} & =\int v_{x^{\prime}}^{(\sigma)} v_{x}^{(\tau)}\left(d x^{\prime}\right), & & x \in X, \sigma, \tau \in \Sigma .
\end{aligned}
$$

If $f \in C(X), \mu \in \operatorname{rca}(X)$, then the extension to $\beta \Sigma$ of the element $(V(\sigma) f, \mu)$, $\sigma \in \Sigma$, of $m(\Sigma)$ is $\mu\langle\dot{\mathfrak{I}}\rangle f$, and if $\hat{\lambda} \in b a(\Sigma)$ corresponds to $\lambda \in \operatorname{rca}(\beta \Sigma)$ then

$$
\int(V(\sigma) f, \mu) \hat{\lambda}(d \sigma)=(f, \lambda\langle\dot{\mathfrak{l}}\rangle \mu) .
$$

It is easy to check that when $\lambda \in L M$ then $\lambda\langle\dot{\mathfrak{l}}\rangle \mu \geqq 0$ if $\mu \geqq 0$ and $(1, \lambda\langle\dot{\mathfrak{l}}\rangle \mu)=(1, \mu)$, $\mu \in \operatorname{rca}(X)$; in particular, if $\mu \in r p(X)$ is a mean then $\lambda\langle\dot{\mathfrak{l}}\rangle \mu$ is a mean. We denote 
by $\operatorname{LM}(X)$ the set of left invariant means for the representation $V^{*}(\sigma)$ on $\operatorname{rca}(X)$. Then for each $\lambda \in L M$ we have $L M(X)=\{\lambda\langle\dot{I}\rangle \mu: \mu \in \operatorname{rp}(X)\}$. That $L M(X)$ is a compact convex set in $r c a(X) \backslash C(X)$ follows from the fact that each $V^{*}(\sigma)$ is an adjoint.

In all that follows it should be kept in mind that when $X$ above is $\beta \Sigma$ and $V(\sigma)$ is the antirepresentation $L^{*}(\sigma)$, then $L M(\beta \Sigma)$ is just the set $L M$ of left invariant means for $\Sigma$.

We now obtain an algebraic characterization of the extreme points of $L M(X)$. As always, it is assumed that $\Sigma$ is left amenable, so that $L M(X)$ is a nonempty compact convex set in $r c a(X) \mid C(X)$.

THEOREM 5. For $\mu \in L M(X)$ to be an extreme point of $L M(X)$ it is necessary and sufficient that for some $\lambda \in L M$ there obtains

$$
\lambda\langle\dot{\mathfrak{l}}\rangle(f \mu)=(f, \mu) \mu \quad \text { for all } f \in C(X),
$$

equivalent to

$$
\lambda\langle\dot{l}\rangle(F \mu)=(\mu, F) \mu \quad \text { for all } F \in C(\alpha X) .
$$

Moreover, when (8)-(9) hold for one $\lambda \in L M$ they hold for all $\lambda \in L M$.

Proof. Recall that $f \mu$ or $F \mu$ in $r c a(X)$ is the measure on $X$ with $f$ or $F$ as generalized Radon-Nikodym derivative with respect to $\mu$. We first show that (8) and (9) are equivalent. Let $\left\{f_{\alpha}\right\}$ be a generalized sequence in $C(X)$ such that $\left\{\kappa f_{\alpha}\right\}$ converges in $C(\alpha X) \mid r c a(X)$ to $F \in C(\alpha X)$. For any $G \in C(\alpha X)$ we have

$$
\begin{aligned}
\left(\lambda\langle\dot{\mathfrak{l}}\rangle\left(f_{\alpha} \mu\right), G\right) & =\left(f_{\alpha} \mu, G\langle\dot{\mathfrak{l}}\rangle \lambda\right) \\
& =\left(\mu,\left(\kappa f_{\alpha}\right)(G\langle\dot{\mathfrak{l}}\rangle \lambda)\right) \\
& \rightarrow(\mu, F(G\langle\dot{\mathfrak{l}}\rangle \lambda)) \\
& =(F \mu, G\langle\dot{\mathfrak{l}}\rangle \lambda) \\
& =(\lambda\langle\dot{\mathfrak{l}}\rangle(F \mu), G) .
\end{aligned}
$$

It follows that (8) implies (9); the converse is vacuous.

Suppose $\mu \in L M(X)$ is not extreme, $\mu=\frac{1}{2} \mu_{1}+\frac{1}{2} \mu_{2}$ with $\mu_{1}, \mu_{2} \in L M(X), \mu_{1} \neq \mu_{2}$. Some version of the Radon-Nikodym derivative $d \mu_{1} / d \mu$ is a Borel function such that $0 \leqq d \mu_{1} / d \mu \leqq 2$. Thus there exists $F \in C(\alpha X)$ such that $\mu_{1}=F \mu$ as an Arens product. For any $\lambda \in L M$ we have $\lambda\langle\dot{\mathrm{I}}\rangle(F \mu)=\lambda\langle\dot{\mathrm{I}}\rangle \mu_{1}=\mu_{1} \neq \mu=(\mu, F) \mu$, whence (9) fails for every $\lambda \in L M$.

Conversely, suppose $\mu \in L M(X)$ is such that (8) fails for some $\lambda \in L M$. Then there exist $\lambda \in L M, f \in C(X)$ such that $\lambda\langle\dot{l}\rangle(f \mu)-(f, \mu) \mu \neq 0$. Consider the signed invariant measure $\nu=\lambda\langle\dot{I}\rangle(f \mu)$, for which $(1, \nu)=(f, \mu)$. If there were a $k$ such that $\nu=k \mu$ then we would have $(1, \nu)=k$ and hence $\lambda\langle\dot{I}\rangle(f \mu)-(f, \mu) \mu=k \mu-k \mu=0$, 
contradicting the choice of $\lambda$ and $f$. Thus $\nu$ is no multiple of $\mu$, zero included. Let $c>0$ be chosen so that $c\|f\|<1$. Then $(1 \pm c f) \mu$ are nonnegative measures, and

$$
\frac{\lambda\langle\dot{\mathfrak{l}}\rangle[(1 \pm c f) \mu]}{1 \pm c(f, \mu)}=\frac{\mu \pm c \nu}{1 \pm c(f, \mu)}
$$

are distinct members of $L M(X)$. It then follows from

$$
\mu=\frac{1+c(f, \mu)}{2} \cdot \frac{\mu+c \nu}{1+c(f, \mu)}+\frac{1-c(f, \mu)}{2} \cdot \frac{\mu-c \nu}{1-c(f, \mu)}
$$

that $\mu$ is not an extreme point of $\operatorname{LM}(X)$.

7. Some properties of the projections. The operator $Q(\lambda) \mu=\lambda\langle\dot{\mathfrak{l}}\rangle \mu, \mu \in \operatorname{rca}(X)$, is a projection onto the left invariant vectors of the representation $V^{*}(\sigma), \sigma \in \Sigma$. The adjoint $Q^{*}(\lambda): C(\alpha X) \rightarrow C(\alpha X)$ can be expressed in terms of Arens operations as $Q^{*}(\lambda) F=F\langle\dot{\mathrm{l}}\rangle \lambda, F \in C(\alpha X)$, and is a nonnegative projection of unit norm in $C(\alpha X)$ such that $Q^{*}(\lambda) 1=1$. Let us apply to $Q^{*}(\lambda)$ the results described in $\S 2$.

We put $q_{\xi}=Q^{* *}(\lambda) \delta_{\xi}, \xi \in \alpha X$, and introduce the equivalence relation such that $q$ is constant on equivalence classes. Let $Y=\left\{q_{\xi}: \xi \in \alpha X\right\}$ be the range of $q$, and let $Y_{0} \subset Y$ be the extreme points of the closed convex hull of $Y$ in $r c a(\alpha X) \mid C(\alpha X)$. The support $\mathscr{S}\left(Q^{*}(\lambda)\right)$ of $Q^{*}(\lambda)$ is $\mathscr{S}\left(Q^{*}(\lambda)\right)=q^{-1} Y_{o}$. If $\xi \in \mathscr{S}\left(Q^{*}(\lambda)\right)$ then $q_{\xi}=y \in Y_{o}$ is a probability measure with closed support contained in the equivalence class $q^{-1} y$. Let a product be defined in range $Q^{*}(\lambda)$ by

$$
Q^{*}(\lambda)\left(Q^{*}(\lambda) F \cdot Q^{*}(\lambda) G\right)
$$

$F, G \in C(\alpha X)$, making range $Q^{*}(\lambda)$ an algebra isomorphic to $C\left(Y_{o}\right)$. Then $\phi \in r p(\alpha X)$ induces a homomorphism of range $Q^{*}(\lambda)$ onto the reals if and only if $\phi$ has closed support in some equivalence class $q^{-1} y$ for some $y \in Y_{o}$; in this event $y=Q^{* *}(\lambda) \phi$ is the element of range $Q^{* *}(\lambda)$ inducing the same homomorphism.

Suppose $\mu \in L M(X)$. Since $\bar{\kappa} Q(\lambda)=Q^{* *}(\lambda) \bar{\kappa}$, we have $Q^{* *}(\lambda) \bar{\kappa} \mu=\bar{\kappa} Q(\lambda) \mu=\bar{\kappa} \mu$, whence $\bar{\kappa} \mu$ is in range $Q^{* *}(\lambda)$. The arguments of Theorem 3 show that

$$
(Q(\lambda)(F \mu), G)=\left(Q^{*}(\lambda)\left(Q^{*}(\lambda) F \cdot Q^{*}(\lambda) G\right), \bar{\kappa} \mu\right)
$$

for all $F, G \in C(\alpha X)$. Suppose $\mu$ is an extreme point of $L M(X)$. Condition (9) of Theorem 5 gives

$$
\left(Q^{*}(\lambda)\left(Q^{*}(\lambda) F \cdot Q^{*}(\lambda) G\right), \bar{\kappa} \mu\right)=\left(Q^{*}(\lambda) F, \bar{\kappa} \mu\right)\left(Q^{*}(\lambda) G, \bar{\kappa} \mu\right) \text { for all } F, G \in C(\alpha X),
$$

which is to say, $\bar{\kappa} \mu \in$ range $Q^{* *}(\lambda)$ induces a homomorphism of range $Q^{*}(\lambda)$. It follows that $\bar{\kappa} \mu \in Y_{o}$, and $q_{\xi}=\bar{\kappa} \mu$ holds on the equivalence class $\xi \in q^{-1} \bar{\kappa} \mu$.

We obtain at once generalizations of some results well known in ergodic theory.

THEOREM 6. If $\mu_{1}$ and $\mu_{2}$ are two distinct extreme points of $L M(X)$ then $\mu_{1}$ and $\mu_{2}$ are mutually singular.

Proof. If the equivalence classes $q^{-1} \bar{\kappa} \mu_{1}$ and $q^{-1} \bar{\kappa} \mu_{2}$ were the same then $q_{\xi}$ $=\bar{\kappa} \mu_{1}=\bar{\kappa} \mu_{2}$ would force $\mu_{1}=\mu_{2}$. Thus $q^{-1} \bar{\kappa} \mu_{1}$ and $q^{-1} \bar{\kappa} \mu_{2}$ are disjoint, $\bar{\kappa} \mu_{1}$ and $\bar{\kappa} \mu_{2}$ have disjoint closed supports, whence $\mu_{1}$ and $\mu_{2}$ are mutually singular. 
THEOREM 7. If $\mu_{1}$ is an extreme point of $\operatorname{LM}(X)$ and if $\mu_{2} \in \operatorname{rca}(X)$ is absolutely continuous with respect to $\mu_{1}$ then $Q(\lambda) \mu_{2}=\left(1, \mu_{2}\right) \mu_{1}$ for every $\lambda \in L M$. In particular, if $\mu_{2}$ is also left invariant then $\mu_{2}=\left(1, \mu_{2}\right) \mu_{1}$.

Proof. If $\mu_{2}$ is absolutely continuous with respect to $\mu_{1}$ then the closed support of $\bar{\kappa} \mu_{2}$ is contained in the closed support of $\bar{\kappa} \mu_{1}$, a fortiori in the equivalence class $q^{-1} \bar{\kappa} \mu_{1}$. Thus on range $Q^{*}(\lambda)$ we have $\left(Q^{*}(\lambda) F, \bar{\kappa} \mu_{2}\right)=\left(1, \bar{\kappa} \mu_{2}\right)\left(Q^{*}(\lambda) F, \bar{\kappa} \mu_{1}\right)$, $F \in C(\alpha X)$, whence $Q(\lambda) \mu_{2}=\left(1, \mu_{2}\right) \mu_{1}$, holding for any $\lambda \in L M$.

It is thus apparent that applying the embedding $\bar{\kappa}$ to $L M(X)$ gives some useful information. Can one get back down? If $\phi \in r p(\alpha X)$ then from

$$
\left(V^{*}(\sigma) Q(\lambda)\right)^{* *}=V^{* * *}(\sigma) Q^{* *}(\lambda)
$$

it follows that $Q^{* *}(\lambda) \phi$ is a left invariant mean on $\alpha X$ for the representation $V^{* * *}(\sigma)$. From $\kappa V(\sigma)=V^{* *}(\sigma) \kappa$ there follows $V^{*}(\sigma) \kappa^{*}=\kappa^{*} V^{* * *}(\sigma)$ and hence $V^{*}(\sigma) \kappa^{*} Q^{* *}(\lambda)=\kappa^{*} Q^{* *}(\lambda)$, which is to say, $\kappa^{*} Q^{* *}(\lambda) \phi \in L M(X)$. If $\mu \in \operatorname{LM}(X)$ then $\kappa^{*} Q^{* *}(\lambda) \bar{\kappa} \mu=\mu$, and it follows that $\kappa^{*} Q^{* *}(\lambda)$ induces a continuous mapping of $r p(\alpha X)$ in $r c a(\alpha X) \backslash C(\alpha X)$ onto $L M(X)$ in $r c a(X) \mid C(X)$.

There are several interesting problems for which this setting may prove useful. First, when is the set of extreme points of $L M(X)$ a compact set in $r c a(X) \backslash C(X)$ ? The elements of $Y_{o}$ are the extreme points of $\left\{Q^{* *}(\lambda) \phi: \phi \in r p(\alpha X)\right\}$, and $\kappa^{*} Y_{o}$ is a compact set in $r c a(X) \backslash C(X)$ containing every extreme point of $L M(X)$. The conjecture that each $\kappa^{*} y, y \in Y_{o}$, is an extreme point of $L M(X)$ is tempting. Second, ergodic sets. The various extreme points $\mu$ of $L M(X)$ live on disjoint open closed sets $\mathscr{S}_{\mu}$ when embedded in $\alpha X$. Is it possible to enclose the $\mathscr{S}_{\mu}$ in disjoint sets of the form $E_{\mu}$ for $E_{\mu} \in X$ ? If so the $E_{\mu}$ are disjoint Borel supports for the various extreme $\mu \in L M(X)$.

Some further properties of $Q^{*}(\lambda)$ are of interest.

THEOREM 8. The support $\mathscr{S}\left(Q^{*}(\lambda)\right)$ is independent of $\lambda \in L M$, for each $\xi \in \mathscr{S}\left(Q^{*}(\lambda)\right)$ the measure $q_{\xi}=Q^{* *}(\lambda) \delta_{\xi}$ is independent of $\lambda \in L M$, and $Y_{0}=\left\{q_{\xi}: \xi \in \mathscr{S}\left(Q^{*}(\lambda)\right)\right\}$ is independent of $\lambda \in L M$.

Proof. If $\lambda_{1}, \lambda_{2} \in L M$ then $Q\left(\lambda_{2}\right) Q\left(\lambda_{1}\right)=Q\left(\lambda_{1}\right)$, whence $Q^{*}\left(\lambda_{1}\right) Q^{*}\left(\lambda_{2}\right)=Q^{*}\left(\lambda_{1}\right)$. Let $Y_{o}^{(2)} \subset$ range $Q^{* *}\left(\lambda_{2}\right)$ be the homomorphisms of range $Q^{*}\left(\lambda_{2}\right)$, so that for each $y \in Y_{o}^{(2)}$ we have $\left(F \cdot Q^{*}\left(\lambda_{2}\right) G, y\right)=(F, y)(G, y)$ for all $F, G \in C(\alpha X)$. From $Q^{* * *}\left(\lambda_{2}\right) y=y$ we obtain

$$
Q^{* *}\left(\lambda_{1}\right) y=Q^{* *}\left(\lambda_{1}\right)\left(Q^{* *}\left(\lambda_{2}\right) y\right)=\left(Q^{* *}\left(\lambda_{1}\right) Q^{* *}\left(\lambda_{2}\right)\right) y=Q^{* *}\left(\lambda_{2}\right) y=y,
$$

whence $y$ is in range $Q^{* *}\left(\lambda_{1}\right)$. For each $y \in Y_{o}^{(2)}$ and all $F, G \in C(\alpha X)$ we have

$$
\begin{aligned}
\left(F \cdot Q^{*}\left(\lambda_{1}\right) G, y\right) & =\left(F \cdot Q^{*}\left(\lambda_{1}\right) Q^{*}\left(\lambda_{2}\right) G, y\right) \\
& =\left(Q^{*}\left(\lambda_{1}\right) F \cdot Q^{*}\left(\lambda_{2}\right) G, y\right) \\
& =(F, y)(G, y)
\end{aligned}
$$


so that $y \in$ range $Q^{* *}\left(\lambda_{1}\right)$ induces a homomorphism of range $Q^{*}\left(\lambda_{1}\right)$. It follows that $y \in Y_{o}^{(1)}$, whence $q_{\xi}^{(1)}=q_{\xi}^{(2)}=y$ on $\left(q^{(1)}\right)^{-1} y=\left(q^{(2)}\right)^{-1} y$. This is to say, $Y_{o}$ is independent of $\lambda \in L M$, and $q_{\xi}=Q^{* *}(\lambda) \delta_{\xi}$ is independent of $\lambda \in L M$ for each

$$
\xi \in \mathscr{S}\left(Q^{*}(\lambda)\right)=q^{-1} Y_{o} .
$$

We will denote by $\mathscr{S}\left(Q^{*}\right)$ this common support of each $Q^{*}(\lambda)$.

Let $\mathscr{M}$ denote the left invariant members of $r c a(X)$. Then $\mathscr{M}=$ range $Q(\lambda)$ for each $\lambda \in L M$, and $\mathscr{M}$ is a Banach space. The Banach space conjugate $\mathscr{M}^{*}$ is isometrically isomorphic to range $Q^{*}(\lambda)$, and hence to $C\left(Y_{o}\right)$. It follows that $Y_{o}$ is hyperstonian, with the members of $\mathscr{M}$ corresponding to the normal measures on $Y_{o}$. The isomorphism of $C\left(Y_{o}\right)$ to range $Q^{*}(\lambda)$ is effected by the injection $\Phi(\lambda): C\left(Y_{o}\right) \rightarrow C(\alpha X)$ given by $(\Phi(\lambda) u)(\xi)=\int u\left(q_{\xi^{\prime}}\right) q_{\xi}\left(d \xi^{\prime}\right), \xi \in \alpha X, u \in C\left(Y_{o}\right)$. The adjoint $\Phi^{*}(\lambda): r c a(\alpha X) \rightarrow r c a\left(Y_{o}\right)$ has the form $\left(\Phi^{*}(\lambda) \theta\right)(F)=\left(Q^{* *}(\lambda) \theta\right)\left(q^{-1} F\right)$, $F \in Y_{o}, \theta \in r c a(\alpha X)$. When $\theta$ has closed support in $\mathscr{S}\left(Q^{*}\right)$ this is simply $\left(\Phi^{*}(\lambda) \theta\right)(F)=\theta\left(q^{-1} F\right), F \in Y_{o}$; that is, one restricts the domain of $\theta$ to unions of equivalence classes $q^{-1} y$. The isomorphism of range $Q^{*}(\lambda)$ onto $C\left(Y_{o}\right)$ is induced by $Q^{\prime}: C(\alpha X) \rightarrow C\left(Y_{o}\right)$ given by $\left(Q^{\prime} F\right)(y)=\left(Q^{*}(\lambda) F\right)(\xi)$ for $y=q_{\xi} \in Y_{o}$. The adjoint $Q^{*}: r c a\left(Y_{o}\right) \rightarrow r c a(\alpha X)$ is $Q^{*} \phi=\int y \phi(d y), \phi \in r c a\left(Y_{o}\right)$; this maps the normal measures on $Y_{o}$ 1-1 onto the normal measures in range $Q^{* *}(\lambda)$.

Let $\mathscr{S}_{o}$ be the closure of $\bigcup\left\{\mathscr{S}_{\mu}: \mu \in L M(X)\right\}$. Then $\mathscr{S}_{o}$ is open in $\alpha X$ and a subset of $\mathscr{S}\left(Q^{*}\right)$, and $\mathscr{S}_{o}$ contains the closed support of each $q_{\xi}, \xi \in \alpha X$. (To see this, let $\lim _{\alpha} \bar{\kappa} \mu_{\alpha}=\delta_{\xi}$ in $r c a(\alpha X) \mid C(\alpha X)$, with $\mu_{\alpha} \in \operatorname{rp}(X)$, so that $\lim _{\alpha} \bar{\kappa} Q(\lambda) \mu_{\alpha}=q_{\xi}$. From $\left(\bar{\kappa} Q(\lambda) \mu_{\alpha}\right)\left(\mathscr{S}_{o}\right)=1$ there follows $q_{\xi}\left(\mathscr{S}_{o}\right)=1$.) The author has not been able to determine whether $\mathscr{S}_{0}=\mathscr{S}\left(Q^{*}\right)$, or even whether $\mathscr{S}_{o}$ is the interior of $\mathscr{S}\left(Q^{*}\right)$. We denote by $\bar{q}$ the restriction of $q: \alpha X \rightarrow Y$ to $\mathscr{S}_{o}$, so that $\bar{q}$ maps $\mathscr{S}_{o}$ onto $Y_{o}$, and $\bar{q}^{-1}$ sends subsets of $Y_{o}$ into subsets of $\mathscr{S}_{o}$. If $\mu \in L M(X)$ then $\Phi^{*}(\lambda) \bar{\kappa} \mu$ is a normal measure on $Y_{o}$, with closed support $\mathscr{T}_{\mu}$ which is open. Since $\bar{\kappa} \mu=Q^{* *} \Phi^{*}(\lambda) \bar{\kappa} \mu$ when $\mu \in L M(X)$, the closed support $\mathscr{S}_{\mu}$ of $\bar{\kappa} \mu$ is contained in $\bar{q}^{-1} \mathscr{T}_{\mu}$, and $\bar{q}^{-1} \mathscr{T}_{\mu}$ is the union of the equivalence classes intersecting $\mathscr{S}_{\mu}$. Later on we shall use the fact that $\mathscr{S}_{\mu}=\bar{q}^{-1} \mathscr{T}_{\mu}$. The argument is as follows. From $\bar{\kappa} \mu=\int q_{\xi} \bar{\kappa} \mu(d \xi)$ it follows that $q_{\xi}\left(\mathscr{S}_{\mu}\right)=1$ for all $\xi \in \mathscr{S}_{\mu}$, and hence for all $\xi \in \bar{q}^{-1} \mathscr{T}_{\mu}$, since $q$ is constant on equivalence classes. We thus have $q_{\xi}\left(\bar{q}^{-1} \mathscr{T}_{\mu}-\mathscr{S}_{\mu}\right)=0$ for all $\xi \in \mathscr{S}_{o}$, using the fact that $q_{\xi}\left(\bar{q}^{-1} \mathscr{T}_{\mu}\right)=0$ if $\xi \in \mathscr{S}_{o}-\bar{q}^{-1} \mathscr{T}_{\mu}$. If the open closed set $\bar{q}^{-1} \mathscr{T}_{\mu}-\mathscr{S}_{\mu}$ in $\mathscr{S}_{o}$ were nonempty, however, then it would assign positive measure to $\bar{\kappa} \nu$ for some $\nu \in L M(X)$, and this would require $q_{\xi}\left(q^{-1} \mathscr{T}_{\mu}-\mathscr{S}_{\mu}\right)>0$ for some $\xi \in \mathscr{S}_{\nu}$. It follows that $\mathscr{S}_{\mu}=\bar{q}^{-1} \mathscr{T}_{\mu}$.

When $\Sigma$ is Abelian there is another projection on $C(\alpha X)$ of interest. Let $\left\{\lambda_{\alpha}\right\}$ be a generalized sequence of means in $l(\Sigma)$ such that $\left\{\kappa_{1} \lambda_{\alpha}\right\}$ converges in $r c a(\beta \Sigma) \mid C(\beta \Sigma)$ to $\lambda \in L M$. Then $V^{* *}\left(\lambda_{\alpha}\right)$ converges in the weak* operator topology to an operator $R(\lambda)$. In terms of Arens operations that is $R(\lambda) F=F\langle\dot{\mathfrak{r}}\rangle \lambda, F \in C(\alpha X)$, and there follows $R\left(\lambda_{1}\right) R\left(\lambda_{2}\right)=R\left(\lambda_{2}\langle\mathfrak{r}\rangle \lambda_{1}\right), \lambda_{1}, \lambda_{2} \in L M$. When $\Sigma$ is Abelian we have $\lambda_{2}\langle\mathfrak{r}\rangle \lambda_{1}=\lambda_{1}\langle\mathfrak{l}\rangle \lambda_{2}$, so that $R\left(\lambda_{1}\right) R\left(\lambda_{2}\right)=R\left(\lambda_{2}\right), \lambda_{1}, \lambda_{2} \in L M$. It is easily verified that 
$Q^{*}\left(\lambda_{1}\right) R\left(\lambda_{2}\right)=Q^{*}\left(\lambda_{1}\right), \lambda_{1}, \lambda_{2} \in L M$, and $Q^{*}(\lambda) \kappa=R(\lambda) \kappa$. We put $r_{\xi}=R^{*}(\lambda) \delta_{\xi}$, $\xi \in \alpha X$. In Theorems 9 and 14-15, following, the assumption that $\Sigma$ is Abelian is made only to insure that $R(\lambda)$ is idempotent.

THEOREM 9. Let $\Sigma$ be Abelian. For each $\lambda \in L M$ we have $r_{\xi}=q_{\xi}$ for all $\xi \in \mathscr{S}\left(Q^{*}\right)$, and $\mathscr{S}\left(Q^{*}\right) \subset \mathscr{S}(R(\lambda))$.

Proof. If $Q^{* *}(\lambda) \phi=\phi$ then

$$
R^{*}(\lambda) \phi=R^{*}(\lambda)\left(Q^{* *}(\lambda) \phi\right)=\left(R^{*}(\lambda) Q^{* *}(\lambda)\right) \phi=Q^{* *}(\lambda) \phi=\phi,
$$

so that $\phi \in$ range $R^{*}(\lambda)$. Suppose $\mu \in L M(X)$. From Theorem 3 we have

$$
R^{*}(\lambda)(F \bar{\kappa} \mu)=(R(\lambda) F) \bar{\kappa} \mu
$$

and hence $\kappa^{*} R^{*}(\lambda)(F \bar{\kappa} \mu)=(R(\lambda) F) \mu$. From $R(\lambda) \kappa=Q^{*}(\lambda) \kappa$ there follows $\kappa^{*} R^{*}(\lambda)$ $=\kappa^{*} Q^{* *}(\lambda)$, and since

$$
\kappa^{*} Q^{* *}(\lambda)(F \bar{\kappa} \mu)=\left(Q^{*}(\lambda) F\right) \mu,
$$

we have $\left(Q^{*}(\lambda) F\right) \mu=(R(\lambda) F) \mu$ for all $F \in C(\alpha X)$, all $\mu \in L M(X)$, whence

$$
\left(Q^{*}(\lambda) F\right) \bar{\kappa} \mu=(R(\lambda) F) \bar{\kappa} \mu
$$

if $\mu \in \operatorname{LM}(X)$. Let $\left\{\bar{\kappa} \mu_{\alpha}\right\}$ be a generalized sequence converging in $r c a(\alpha X) \backslash C(\alpha X)$ to $\delta_{\xi}, \xi \in \alpha X$, so that $\left\{Q^{* *}(\lambda) \bar{\kappa} \mu_{\alpha}\right\}=\left\{\bar{\kappa} Q(\lambda) \mu_{\alpha}\right\}$ converges in $r c a(\alpha X) \backslash C(\alpha X)$ to $Q^{* *}(\lambda) \delta_{\xi}=q_{\xi}$. From the relation above we obtain $\left(Q^{*}(\lambda) F\right) q_{\xi}=(R(\lambda) F) q_{\xi}, \xi \in \alpha X$, and it is straightforward that $Q^{*}(\lambda)(G \cdot R(\lambda) F)=Q^{*}(\lambda)\left(G \cdot Q^{*}(\lambda) F\right)$ holds for all $F, G \in C(\alpha X)$. Let $Y_{o}$ be the homomorphisms of range $Q^{*}(\lambda)$. It has been shown that if $y \in Y_{o}$ then $R^{*}(\lambda) y=y$, and we have

$$
\begin{aligned}
(F \cdot R(\lambda) G, y) & =\left(Q^{*}(\lambda)(F \cdot R(\lambda) G), y\right) \\
& =\left(Q^{*}(\lambda)\left(F \cdot Q^{*}(\lambda) G\right), y\right) \\
& =(F, y)(G, y),
\end{aligned}
$$

so that $y$ is a homomorphism of range $R(\lambda)$. Thus for $\xi \in \mathscr{S}\left(Q^{*}\right)$ we have $q_{\xi}=r_{\xi}=y$ on $q^{-1} y=r^{-1} y$, and $\mathscr{S}\left(Q^{*}\right) \subset \mathscr{S}(R(\lambda))$.

8. Left almost convergence. As before, we assume that $\Sigma$ is left amenable, and we assume given an antirepresentation $V(\sigma): C(X) \rightarrow C(X)$ such that $V(\sigma) \geqq 0$, $V(\sigma) 1=1$. We say that $f \in C(X)$ is left almost convergent to value $k$ provided $(f, \mu)=k$ for every $\mu \in L M(X)$. When $X=\beta \Sigma$ and $V(\sigma)=L^{*}(\sigma)$ we extend the notion slightly; an element of $m(\Sigma)$ is left almost convergent to $k$ if its continuous extension to $\beta \Sigma$ is left almost convergent to $k$.

THEOREM 10. For each $\mu \in L M(X)$ and each $f, g \in C(X)$ the element $(f \cdot V(\sigma) g, \mu)$, $\sigma \in \Sigma$, of $m(\Sigma)$ is left almost convergent to value $\left(g,\left(Q^{*}(\lambda) \kappa f\right) \mu\right)=\left(f,\left(Q^{*}(\lambda) \kappa g\right) \mu\right)$, which value is independent of $\lambda \in L M$. For $\mu \in L M(X)$ to be an extreme point of $\operatorname{LM}(X)$ it is necessary and sufficient that this value be $(f, \mu)(g, \mu)$ for all $f, g \in C(X)$. 
Proof. If $Q^{* *}(\lambda) \phi=\phi$ then $Q^{* *}(\lambda)(F \phi)=\left(Q^{*}(\lambda) F\right) \phi, F \in C(\alpha X)$, by Theorem 3 . Now, such a $\phi$ has closed support in $\mathscr{S}\left(Q^{*}\right)$, and if $\xi \in S\left(Q^{*}\right)$ then

$$
\left(Q^{*}(\lambda) F\right)(\xi)=\int F\left(\xi^{\prime}\right) q_{\xi}\left(d \xi^{\prime}\right)
$$

is independent of $\lambda \in L M$, by Theorem 8. Thus $Q^{* *}(\lambda)(F \phi)$ is independent of $\lambda \in L M$ when $Q^{* *}(\lambda) \phi=\phi$. If $f, g \in C(X)$ then

$$
\begin{aligned}
\int(f \cdot V(\sigma) g, \mu) \hat{\lambda}(d \sigma) & =(g, Q(\lambda)(f \mu))=\left(\kappa g, Q^{* *}(\lambda)(\kappa f \bar{\kappa} \mu)\right) \\
& =\left(\kappa g,\left(Q^{*}(\lambda) \kappa f\right) \bar{\kappa} \mu\right)=\left(g,\left(Q^{*}(\lambda) \kappa f\right) \mu\right)
\end{aligned}
$$

is independent of $\lambda \in L M$, which is to say, $(f \cdot V(\sigma) g, \mu), \sigma \in \Sigma$, is left almost convergent to $\left(g,\left(Q^{*}(\lambda) \kappa f\right) \mu\right)$, this value being independent of $\lambda \in L M$. Applying condition (8) of Theorem 5, we see that for $\mu \in L M(X)$ to be extreme it is necessary and sufficient that this value be $(f, \mu)(g, \mu)$ for all $f, g \in C(X)$. This condition is equivalent to $\left(Q^{*}(\lambda) \kappa f\right) \mu=(f, \mu) \mu$, and is simply the assertion that $Q^{*}(\lambda) \kappa f$ is the constant $(f, \mu)$ on $\mathscr{S}_{\mu}$.

Let us examine the notion of left almost convergence in more detail. If $\lambda \in L M$ is fixed then $\{\lambda\langle\dot{\mathfrak{l}}\rangle \mu: \mu \in \operatorname{rp}(X)\}$ is the whole of $L M(X)$. Thus $f \in C(X)$ is left almost convergent to value $k$ provided $(f, \lambda\langle\dot{\mathfrak{l}}\rangle \mu)=(\mu\langle\dot{\mathfrak{l}}\rangle f, \lambda)=k$ for all $\mu \in \operatorname{rp}(X)$. Let $\left\{\lambda_{\alpha}\right\}$ be a generalized sequence of means in $l(\Sigma)$ such that $\left\{\kappa_{1} \lambda_{\alpha}\right\}$ converges in $\operatorname{rca}(\beta \Sigma) \backslash C(\beta \Sigma)$ to $\lambda \in L M$. Then

$$
\begin{aligned}
(\mu\langle\dot{\mathfrak{l}}\rangle f, \lambda) & =\lim _{\alpha}\left(\mu\langle\dot{\mathfrak{l}}\rangle f, \kappa_{1} \lambda_{\alpha}\right) \\
& =\lim _{\alpha}\left(\lambda_{\alpha}, \mu\langle\dot{\mathfrak{l}}\rangle f\right) \\
& =\lim _{\alpha}\left(f * \lambda_{\alpha}, \mu\right)
\end{aligned}
$$

where

$$
f * \lambda_{\alpha}=\sum_{\sigma} \lambda_{\sigma}^{(\alpha)} V(\sigma) f
$$

is the right module product in terms of which the Arens operations are defined. One sees from (10) that $\left\{f * \lambda_{\alpha}\right\}$ is always a weak generalized Cauchy sequence, and that for $f$ to be left almost convergent to value $k$ it is necessary and sufficient that $\left\{f * \lambda_{\alpha}\right\}$ converge in $C(X) \mid r c a(X)$ to the constant function $k \in C(X)$.

Fix $\lambda \in L M$ and a generalized sequence $\left\{\lambda_{\alpha}\right\}$ of means in $l(\Sigma)$ such that $\left\{\kappa_{1} \lambda_{\alpha}\right\}$ converges in $r c a(\beta \Sigma) \mid C(\beta \Sigma)$ to $\lambda$. Let $\mathscr{D}_{\lambda}$ be the linear subspace of $C(X)$ defined by $\mathscr{D}_{\lambda}=\left\{f \in C(X): f * \lambda_{\alpha}\right.$ converges weakly to an element of $\left.C(X)\right\}$. If $f \in \mathscr{D}_{\lambda}$ let $S_{\lambda} f \in C(X)$ denote the weak limit of $\left\{f * \lambda_{\alpha}\right\}$. We see from (10) that if $f \in \mathscr{D}_{\lambda}$ then 
$\left(S_{\lambda} f, \mu\right)=(f, Q(\lambda) \mu)=\left(\mu, Q^{*}(\lambda) \kappa f\right)$ for all $\mu \in r c a(X)$, whence $Q^{*}(\lambda) \kappa f=\kappa S_{\lambda} f$. Conversely, suppose $f \in C(X)$ is such that $Q^{*}(\lambda) \kappa f=\kappa g$ for some $g \in C(X)$. Then for all $\mu \in \operatorname{rca}(X)$ we have

$$
\begin{aligned}
(g, \mu) & =(\mu, \kappa g) \\
& =\left(\mu, Q^{*}(\lambda) \kappa f\right) \\
& =(f, Q(\lambda) \mu) \\
& =\lim _{\alpha}\left(f, \kappa_{1} \lambda_{\alpha}<\dot{\mathfrak{l}}>\mu\right) \\
& =\lim _{\alpha}\left(f * \lambda_{\alpha}, \mu\right),
\end{aligned}
$$

whence $f \in \mathscr{D}_{\lambda}$ and $g=S_{\lambda} f$. Thus $\mathscr{D}_{\lambda}=\left\{f \in C(X): Q^{*}(\lambda) \kappa f \in \kappa C(X)\right\}$. It follows that $\mathscr{D}_{\lambda}$ is norm closed in $C(X)$, and that $\mathscr{D}_{\lambda}$ does not depend on the particular generalized sequence $\left\{\lambda_{\alpha}\right\}$ used to approximate $\lambda$. Thus $f \in C(X)$ is left almost convergent to $k$ if and only if for some $\lambda \in L M$ there obtains $f \in \mathscr{D}_{\lambda}$ and $S_{\lambda} f=k$; in this event $f \in \mathscr{D}_{\lambda}$ and $S_{\lambda} f=k$ holds for every $\lambda \in L M$.

The considerations above resemble those of the Eberlein-Day ergodic theorem [1]; the elements of $\mathscr{D}_{\lambda}$ could be called left ergodic members of $C(X)$ for $\lambda$. Our results cannot be obtained from the Eberlein-Day theorem, however, because we have only one sided invariance. Nevertheless, we are able to obtain strong convergence to $k$ if the generalized sequence $\left\{\lambda_{\alpha}\right\}$ is appropriately chosen. Fix $\lambda \in L M$, and let $\left\{\lambda_{\alpha}\right\}$ be a generalized sequence of means in $l(\Sigma)$ such that $\lim _{\alpha} \kappa_{1} \lambda_{\alpha}=\lambda$ in $r c a(\beta \Sigma) \mid C(\beta \Sigma)$ and $\lim _{\alpha}\left\|\delta_{\sigma} * \lambda_{\alpha}-\lambda_{\alpha}\right\|=0$ for each $\sigma \in \Sigma$. That is, $\left\{\kappa_{1} \lambda_{\alpha}\right\}$ converges weakly* to $\lambda \in L M$ and $\left\{\lambda_{\alpha}\right\}$ converges in norm to left invariance. Proof that such generalized sequences exist is given by Day [1].

THEOREM 11. For $f \in C(X)$ to be left almost convergent to $k$ it is necessary and sufficient that $\lim _{\alpha}\left\|f * \lambda_{\alpha}-k\right\|=0$ for each such generalized sequence $\left\{\lambda_{\alpha}\right\}$.

Proof. If $\left\{f * \lambda_{\alpha}\right\}$ converges in norm to $k$ then it converges weakly and the previous considerations apply. For $f \in C(X)$ define

$$
p(f)=\lim _{\alpha} \sup \max _{x \in X}\left(f * \lambda_{\alpha}\right)(x),
$$

so that

$$
-p(-f)=\liminf _{\alpha} \min _{x \in X}\left(f * \lambda_{\alpha}\right)(x) .
$$

Then $p(f) \geqq-p(-f)$, and clearly, for $\lim _{\alpha}\left\|f * \lambda_{\alpha}-k\right\|=0$ to hold it is necessary and sufficient that $p(f)=-p(-f)$, the common value being $k$. Now, $p(f)$ has the properties $p(f+g) \leqq p(f)+p(g)$ and $p(c f)=c p(f)$ for $c \geqq 0, f, g \in C(X)$. Moreover, $\tilde{p}(f) \geqq 0$ if $f \geqq 0, \tilde{p}(1)=1$, and $|\tilde{p}(f)| \leqq\|f\|, f \in C(X)$, where $\tilde{p}(f)$ is either $p(f)$ or 
$-p(-f)$. It follows that if $\mu \in r c a(X)$ is such that $(f, \mu) \leqq p(f), f \in C(X)$ (HahnBanach) then $\mu \in r p(X)$. We have further, for all $\sigma \in \Sigma$,

$$
\begin{aligned}
\tilde{p}(V(\sigma) f-f) & \leqq \limsup _{\alpha}\left\|f * \delta_{\sigma} * \lambda_{\alpha}-f * \lambda_{\alpha}\right\| \\
& \leqq \limsup _{\alpha}\|f\|\left\|\delta_{\sigma} * \lambda_{\alpha}-\lambda_{\alpha}\right\| \\
& =0
\end{aligned}
$$

and it follows easily that if $(f, \mu) \leqq p(f), f \in C(X)$, then $\mu \in L M(X)$. Suppose then that $g \in C(X)$ is such that $p(g)>-p(-g)$. Then there exist $\mu_{1}, \mu_{2} \in r c a(X)$ such that

$$
\begin{aligned}
\left(f, \mu_{1}\right) & \leqq p(f) \quad \text { for all } f \in C(X), \\
\left(g, \mu_{1}\right) & =p(g), \\
\left(f, \mu_{2}\right) & \leqq p(f) \quad \text { for all } f \in C(X), \\
\left(-g, \mu_{2}\right) & =p(-g) .
\end{aligned}
$$

By what has been said above, $\mu_{1}, \mu_{2} \in L M(X)$ and $\left(g, \mu_{1}\right)>\left(g, \mu_{2}\right)$, so that $g$ is not left almost convergent.

Closely related to Theorem 11 is the following generalization of a result of Jerison [14]. Again, $\left\{\lambda_{\alpha}\right\}$ is any generalized sequence of means in $l(\Sigma)$ such that $\left\{\kappa_{1} \lambda_{\alpha}\right\}$ converges in $\operatorname{rca}(\beta \Sigma) \mid C(\beta \Sigma)$ to $\lambda \in L M$ and $\left\{\lambda_{\alpha}\right\}$ converges in norm to left invariance.

THEOREM 12. If $\mu$ is any extreme point of $L M(X)$ then there is a generalized sequence of the form $\left\{\kappa_{1} \lambda_{\alpha_{y}}\langle\mathfrak{l}\rangle \delta_{x_{y}}\right\}$ converging in $\operatorname{rca}(X) \mid C(X)$ to $\mu$, where $x_{y} \in X$ and $\left\{\lambda_{\alpha_{\gamma}}\right\}$ is a generalized subsequence of $\left\{\lambda_{\alpha}\right\}$.

Proof. Put

$$
\begin{aligned}
A_{\alpha} & =\left\{\kappa_{1} \lambda_{\alpha}\langle\dot{\mathfrak{l}}\rangle \delta_{x}: x \in X\right\}, \\
B_{\alpha} & =\text { closure of } \bigcup_{\beta>\alpha} A_{\beta} \text { in } \operatorname{rca}(X) \mid C(X), \\
B & =\bigcap_{\alpha} B_{\alpha},
\end{aligned}
$$

so that $B$ is the topological limit superior [15] of the generalized sequence of sets $\left\{A_{\alpha}\right\}$. It is clear that the elements of $B$ consist of all limits of convergent generalized sequences of the form $\left\{\kappa_{1} \lambda_{\alpha_{y}}\langle\dot{I}\rangle \delta_{x_{y}}\right\}$ in $\operatorname{rca}(X) \mid C(X)$, with $\left\{\lambda_{\alpha_{y}}\right\}$ a generalized subsequence of $\left\{\lambda_{\alpha}\right\}$. From

$$
\left\|\delta_{\sigma}\langle\dot{\mathfrak{l}}\rangle\left(\kappa_{1} \lambda_{\alpha_{\gamma}}\langle\dot{\mathfrak{l}}\rangle \delta_{x_{y}}\right)-\left(\kappa_{1} \lambda_{\alpha_{y}}\langle\dot{\mathfrak{l}}\rangle \delta_{x_{y}}\right)\right\| \leqq\left\|\delta_{\sigma} * \lambda_{\alpha_{\gamma}}-\lambda_{\alpha_{y}}\right\| \rightarrow 0, \quad \sigma \in \Sigma,
$$

it follows that $B \subset L M(X)$. Let $\overline{c o} B$ denote the closed convex hull of $B$ in 
$r c a(X) \mid C(X)$. If we can show that $\overline{c o} B=L M(X)$ then the arguments of Krein and Milman apply and the theorem is established. First, from $\left(f, \kappa_{1} \lambda_{\alpha}\langle\dot{I}\rangle \mu\right)=\left(f * \lambda_{\alpha}, \mu\right)$, $f \in C(X), \mu \in \operatorname{rca}(X)$, it follows that for each fixed $\alpha$ the function $\kappa_{1} \lambda_{\alpha}\langle\dot{\mathrm{I}}\rangle \mu$ is a continuous function of $\mu$ in $r c a(X) \mid C(X)$. Thus

$$
\begin{aligned}
\overline{\mathrm{co}} A_{\alpha} & =\kappa_{1} \lambda_{\alpha}\langle\dot{\mathfrak{l}}\rangle \overline{\operatorname{co}}\left\{\delta_{x}: x \in X\right\} \\
& =\kappa_{1} \lambda_{\alpha}\langle\dot{\mathfrak{l}}\rangle\{r p(X)\} \\
& \supset L M(X),
\end{aligned}
$$

whence $\overline{c o} B_{\alpha} \supset L M(X)$ for each $\alpha$. It is a consequence of a theorem of Jerison [15] that $\overline{\text { co }} \bigcap_{\alpha} B_{\alpha}=\bigcap_{\alpha} \overline{c o} B_{\alpha}$, so we obtain $\overline{c o} B \supset L M(X)$ as desired.

When $\Sigma$ is the additive positive integers there is another result of Jerison that can be strengthened and generalized. The familiar sequence $\left\{\lambda_{n}\right\}=\left\{\left(\delta_{1}+\cdots+\delta_{n}\right) / n\right\}$ in $l(\Sigma)$ converges in norm to (left) invariance. Let $\Lambda \subset L M$ be the set of limit points of $\left\{\kappa_{1} \lambda_{n}\right\}$ in $r c a(\beta \Sigma) \backslash C(\beta \Sigma)$.

THEOREM 13. For each $\lambda . \in \Lambda$, the closure of the set $\left\{\lambda\langle\dot{I}\rangle \delta_{x}: x \in X\right\}$ in $r c a(X) \backslash C(X)$ contains every extreme point of $L M(X)$.

Proof. The proof follows exactly that of Theorem 3 of [14]; we omit the details. Jerison deals with $\left\{\lambda\langle\dot{l}\rangle \delta_{x}: x \in X, \lambda \in \Lambda\right\}$, but it is not necessary that $\lambda$ vary.

Theorem 13 has an interesting corollary. Let $\left\{\lambda_{n_{\alpha}}\right\}$ be a generalized subsequence of the above sequence $\left\{\lambda_{n}\right\}$ such that $\left\{\kappa_{1} \lambda_{n_{\alpha}}\right\}$ converges in $\operatorname{rca}(\beta \Sigma) \backslash C(\beta \Sigma)$ to $\lambda \in \Lambda$. Then for each $f \in C(X)$ the generalized sequence $\left\{f * \lambda_{n_{\alpha}}\right\}$ converges pointwise on $X$. Suppose $f$ is such that the limit function is the constant function $k$. Then $f$ is (left) almost convergent to $k$, and the sequence $\left\{f * \lambda_{n}\right\}$ converges to $k$ uniformly on $X$. To see this, define

$$
\begin{aligned}
p_{1}(f) & =\sup _{x \in X}\left(f, \lambda\langle\dot{\mathfrak{l}}\rangle \delta_{x}\right) \\
& =\sup _{x \in X} \lim _{\alpha}\left(f * \lambda_{n_{\alpha}}\right)(x), \quad f \in C(X) .
\end{aligned}
$$

Clearly, for $p_{1}(f)=-p_{1}(-f)$ to hold it is necessary and sufficient that $\left\{f * \lambda_{n_{\alpha}}\right\}$ converge pointwise to $k=p_{1}(f)$. By Theorem 13, however,

$$
p_{1}(f)=\sup _{\mu \in L M(X)}(f, \mu),
$$

so if $p_{1}(f)=-p_{1}(-f)=k$ then $f$ is left almost convergent to $k$. The arguments for $\left\{f * \lambda_{n}\right\}$ involve

$$
\lim \sup _{n} \sup _{x \in X}\left(f * \lambda_{n}\right)(x),
$$

and are similar to the usual ones for almost convergence on $\Sigma$. 
In the following, $\left\{\lambda_{\alpha}\right\}$ is any generalized sequence of means in $l(\Sigma)$ such that $\left\{\kappa_{1} \lambda_{\alpha}\right\}$ converges in $\operatorname{rca}(\beta \Sigma) \mid C(\beta \Sigma)$ to $\lambda \in L M$. It is not required that $\left\{\lambda_{\alpha}\right\}$ converge in norm to left invariance.

THEOREM 14. Let $\Sigma$ be Abelian. Then the following conditions on $\mu \in \operatorname{rca}(X)$ are equivalent:

$$
\lim _{\alpha} V^{*}\left(\lambda_{\alpha}\right) \mu=Q(\lambda) \mu \text { weakly in } \operatorname{rca}(X),
$$

$$
R^{*}(\lambda) \bar{\kappa} \mu \in \bar{\kappa} r c a(X) \text {, the value necessarily being } \bar{\kappa} Q(\lambda) \mu \text {. }
$$

When these conditions hold then the set function defined by $\lim _{\alpha}\left(V^{*}\left(\lambda_{\alpha}\right) \mu\right)(E)$, $E \in X$, is countably additive.

Proof. For all $F \in C(\alpha X)$ we have

$$
\lim _{\alpha}\left(V^{*}\left(\lambda_{\alpha}\right) \mu, F\right)=\lim _{\alpha}\left(\mu, V^{* *}\left(\lambda_{\alpha}\right) F\right)=(\mu, R(\lambda) F)=\left(F, R^{*}(\lambda) \bar{\kappa} \mu\right),
$$

according to the definition of the projection $R(\lambda)$. When (11) holds the limit is also $(Q(\lambda) \mu, F)=(F, \bar{\kappa} Q(\lambda) \mu)$, whence $R^{*}(\lambda) \bar{\kappa} \mu=\bar{\kappa} Q(\lambda) \mu$. Conversely, suppose $R^{*}(\lambda) \bar{\kappa} \mu$ $=\bar{\kappa} \nu$ for some $\nu \in \operatorname{rca}(X)$. Then $\nu$ must have the value

$$
\nu=\kappa^{*} \bar{\kappa} \nu=\kappa^{*} R^{*}(\lambda) \bar{\kappa} \mu=\kappa^{*} Q^{* *}(\lambda) \bar{\kappa} \mu=Q(\lambda) \mu .
$$

For each $F \in C(X)$ we have then

$$
\begin{aligned}
\lim _{\alpha}\left(V^{*}\left(\lambda_{\alpha}\right) \mu, F\right) & =\lim _{\alpha}\left(\mu, V^{* *}\left(\lambda_{\alpha}\right) F\right)=(\mu, R(\lambda) F)=\left(F, R^{*}(\lambda) \bar{\kappa} \mu\right) \\
& =(F, \bar{\kappa} Q(\lambda) \mu)=(Q(\lambda) \mu, F),
\end{aligned}
$$

so that (11) holds. Suppose that (11) holds. Then for each $E \in X$ we have

$$
\lim _{\alpha}\left(V^{*}\left(\lambda_{\alpha}\right) \mu\right)(E)=\lim _{\alpha}\left(V^{*}\left(\lambda_{\alpha}\right) \mu, \chi_{E^{\wedge}}\right)=\left(Q(\lambda) \mu, \chi_{E^{\wedge}}\right)=(Q(\lambda) \mu)(E),
$$

and this is countably additive as a function of $E \in \boldsymbol{X}$.

For our final result we recall that $\mathscr{S}_{o}=\bigcup\left\{\mathscr{S}_{\mu}: \mu \in L M(X)\right\}$ is the closed support of the measures in range $Q^{* *}(\lambda)$, and that $\bar{q}^{-1}$ sends subsets of $Y_{o}$ into subsets of $\mathscr{S}_{0}$.

THEOREM 15. For $\mu \in r c a(X)$ to be absolutely continuous with respect to a member of $L M(X)$ it is necessary and sufficient that $\mathscr{S}_{\mu} \subset \mathscr{S}_{o}$. When $\mathscr{S}_{\mu} \subset \mathscr{S}_{o}$ holds then $Q(\lambda) \mu=\nu$ is independent of $\lambda \in L M$ and $\mu$ is $\nu$-continuous. For $\mu \in \operatorname{rca}(X)$ to be equivalent to a member of $L M(X)$ it is necessary and sufficient that $\mathscr{S}_{\mu}$ be a union of equivalence classes $\bar{q}^{-1} y$. When $\Sigma$ is Abelian and $\mathscr{S}_{\mu} \subset \mathscr{S}_{o}$, then conditions (11) and (12) of Theorem 15 are satisfied, and also

For each $E \in X$ the element $\left(V^{*}(\sigma) \mu\right)(E), \sigma \in \Sigma$, of $m(\Sigma)$ is left almost convergent to value $\nu(E)$. 
Proof. We may as well assume $\mu \in r p(X)$. For $\mu$ to be absolutely continuous with respect to a member of $L M(X)$ it is necessary that $\mathscr{S}_{\mu} \subset \mathscr{S}_{o}$, clearly. Suppose that $\mathscr{S}_{\mu} \subset \mathscr{S}_{o}$ holds. Applying Theorem 8 to the representation

$$
\bar{\kappa} Q(\lambda) \mu=\int q_{\xi} \bar{\kappa} \mu(d \xi),
$$

we see that $Q(\lambda) \mu=\nu$ is independent of $\lambda \in L M$. From $\Phi^{*}(\lambda) \bar{\kappa} \mu=\Phi^{*}(\lambda) \bar{\kappa} \nu$ it follows that $\mathscr{S}_{\mu} \subset \bar{q}^{-1} \mathscr{T}_{\nu}$. We showed previously that $\bar{q}^{-1} \mathscr{T}_{\nu}=\mathscr{S}_{v}$, so that $\mathscr{S}_{\mu} \subset \mathscr{S}_{\nu}$ and $\mu$ is $\nu$-continuous. For $\mu$ to be equivalent to $\nu$ it is necessary and sufficient that $\mathscr{S}_{\mu}=\bar{q}^{-1} \mathscr{T}_{\nu}$. Suppose $\mathscr{S}_{\mu} \subset \mathscr{S}_{o}$ and $\Sigma$ is Abelian. Applying Theorem 9 to

$$
\begin{aligned}
R^{*}(\lambda) \bar{\kappa} \mu & =\int r_{\xi} \bar{\kappa} \mu(d \xi), \\
Q^{* *}(\lambda) \bar{\kappa} \mu & =\int q_{\xi} \bar{\kappa} \mu(d \xi),
\end{aligned}
$$

we obtain $R^{*}(\lambda) \bar{\kappa} \mu=\bar{\kappa} Q(\lambda) \mu$, so that conditions (11) and (12) are satisfied. Since for each $E \in X$ we have $\lim _{\alpha}\left(V^{*}\left(\lambda_{\alpha}\right) \mu\right)(E)=\nu(E)$ independent of $\lambda \in L M$, it must be the case that $\left(V^{*}(\sigma) \mu\right)(E), \sigma \in \Sigma$, is left almost convergent to value $\nu(E)$.

9. Right and two sided invariance. When $\Sigma$ is right amenable or (two sided) amenable there are results corresponding in detail to those of $\$ \$ 6-8$. The easiest way to show this is to reduce the right and two sided cases to the left case.

Suppose $\Sigma$ is right amenable. Let $\Sigma^{t}$ be the transposed semigroup of $\Sigma$, i.e., products are reversed, so that $\Sigma^{t}$ is left amenable [1]. Let $U(\sigma): C(X) \rightarrow C(X)$ be a representation of $\Sigma$ such that $U(\sigma) \geqq 0, U(\sigma) 1=1$. Then $U(\sigma), \sigma \in \Sigma^{t}$, is an antirepresentation of left amenable $\Sigma^{t}$, and $\$ \S 6-8$ apply. The left invariant means on $X$ for $\Sigma^{t}$ are the right invariant means $R M(X)$ for the antirepresentation $U^{*}(\sigma)$ of $\Sigma$. We omit the list of theorems corresponding to Theorems 5-15.

Suppose $\Sigma$ is amenable. Let $\Sigma_{1}$ be $\Sigma$ augmented by an identity (if $\Sigma$ does not have one), and let $\Sigma_{2}$ be the product semigroup $\Sigma_{2}=\Sigma_{1} \times \Sigma_{1}^{t}$. The amenability of $\Sigma$ implies amenability of $\Sigma_{2}$ [1]; a fortiori, $\Sigma_{2}$ is left amenable. Assume given a representation $U(\sigma): C(X) \rightarrow C(X)$ and also an antirepresentation

$$
V(\sigma): C(X) \rightarrow C(X)
$$

of $\Sigma$ by transition probabilities, as before. It is required that these commute: $U(\sigma) V(\tau)=V(\tau) U(\sigma), \sigma, \tau \in \Sigma$. We require also that $U(1)=V(1)=1$ for the identity of $\Sigma_{1}$. Then $U(\sigma) V(\tau),(\tau, \sigma) \in \Sigma_{2}$, is an antirepresentation of left amenable $\Sigma_{2}$ by transition probabilities. If $L M(X)$ are the left invariant means of the representation $V^{*}(\sigma)$ of $\Sigma$ and $R M(X)$ are the right invariant means of the antirepresentation $U^{*}(\sigma)$ of $\Sigma$, then it is easy to see that the two sided means $M(X)=L M(X) \cap R M(X)$ are the left invariant means for the representation $U^{*}(\sigma) V^{*}(\tau),(\tau, \sigma) \in \Sigma_{2}$, of left amenable $\Sigma_{2}$, and $\$ 6-8$ apply. In particular, when $X$ is $\beta \Sigma, U(\sigma)$ is $R^{*}(\sigma), V(\tau)$ is 
$L^{*}(\tau)$, then all conditions are satisfied, and $M(\beta \Sigma)$ is the set of two sided means for $\Sigma$. We omit the list of theorems corresponding to Theorems 5-15.

Notes added in proof. I. A further reference for $\$ 2$ is [16]. II. The arguments in Theorems 8-9 are incomplete. In Theorems 8-9 and wherever they are used, $\mathscr{S}\left(Q^{*}(\lambda)\right)$ should be replaced by $\mathscr{S}_{0}$. III. In Theorems 9 and $14-15$, the condition " $\Sigma$ is Abelian" can be replaced by " $\Sigma$ is amenable." IV. The work of L. Sucheston was the inspiration for Theorem 15.

\section{REFERENCES}

1. M. M. Day, Amenable semigroups, Illinois J. Math 1 (1957), 509-544.

2. H. A. Dye, On the ergodic mixing theorem, Trans. Amer. Math. Soc. 118 (1965), 123-130.

3. L. Sucheston, An ergodic application of almost convergent sequences, Duke Math. J. 30 (1963), 417-422.

4. N. Dunford and J. T. Schwartz, Linear operators, Part 1, Interscience, New York, 1958.

5. S. P. Lloyd, On certain projections in spaces of continuous functions, Pacific J. Math. 13 (1963), 171-175.

6. D. A. Edwards, On the representation of certain functionals by measures on the Choquet boundary, Ann. Inst. Fourier (Grenoble) 13 (1963), 111-121.

7. Richard Arens, Operations induced in function classes, Monatsh. Math. 55 (1951), 1-19.

8. - The adjoint of a bilinear operation, Proc. Amer. Math. Soc. 2 (1951), 839-848.

9. Paul Civin and Bertram Yood, The second conjugate space of a Banach algebra as an algebra, Pacific J. Math. 11 (1961), 847-870.

10. S. L. Gulick, The bidual of a locally multiplicatively-convex algebra, Pacific J. Math. 17 (1966), 71-96.

11. D. A. Edwards, A class of topological Boolean algebras, Proc. London Math. Soc. (3) 13 (1963), 413-429.

12. Paul R. Halmos, The range of a vector measure, Bull. Amer. Math. Soc. 54 (1948), 416-421.

13. Mahlon M. Day, Fixed-point theorems for compact convex sets, Illinois J. Math. 5 (1961), 585-590.

14. Meyer Jerison, The set of all generalized limits of bounded sequences, Canad. J. Math. 9 (1957), 79-89.

15. - A property of extreme points of compact convex sets, Proc. Amer. Math. Soc. 5 (1954), 782-783.

16. G. L. Seever, Nonnegative projections on $C_{0}(X)$, Pacific J. Math. 17 (1966), 159-166.

Bell Telephone Laboratories, InCorporated, Murray Hill, New Jersey 